4 Hanyu Rao ${ }^{1,2^{*}}$, Xuefeng $\mathrm{Li}^{1,3^{*}}$, Min $\mathrm{Liu}^{1,2}$, Jing $\mathrm{Liu}^{1,2}$, Xiaoxue $\mathrm{Li}^{1,2}, \mathrm{Jin} \mathrm{Xu}^{2}, \mathrm{Li} \mathrm{Li}^{1,2 \#}$, Wei-Qiang $5 \mathrm{Gao}^{1,2 \#}$

6

\section{Di-Ras2 Promotes Renal Cell Carcinoma Formation by Activating the MAPK Pathway in} the Absence of VHL

\footnotetext{
${ }^{1}$ State Key Laboratory of Oncogenes and Related Genes, Renji-Med X Clinical Stem Cell
} Research Center, Ren Ji Hospital, School of Medicine and School of Biomedical Engineering, Shanghai Jiao Tong University, Shanghai, 200127, China.

${ }^{2}$ Med-X Research Institute, Shanghai Jiao Tong University, Shanghai, China.

3 The First Affiliated Hospital of University of South China, Hengyang, Hunan 421001, P.R. China, Department of Metabolism and Endocrinology, The First Affiliated Hospital of University of South China, Hengyang, Hunan 421001, P.R. China.

Running title: Di-Ras2 activates MAPK pathway in RCC.

Keywords: Di-Ras2; VHL; Ras/MAPK; renal cell carcinoma; cell proliferation.

Article category: Molecular Cancer Biology.

Abbreviations: RCC: renal cell carcinoma; ccRCC: clear cell renal cell carcinoma; TCGA: The Cancer Genome Atlas; KIRC: Kidney clear cell carcinoma; q-PCR: Quantitative PCR; WB: western blot; Co-IP: co-immunoprecipitation; CHX: cycloheximide; MAPK: Mitogen-Activated Protein Kinase Pathway; VHL: von Hippel-Lindau.

Novelty and impact: Our findings have demonstrated the biological and clinical significance of Di-Ras2 as a potential oncogene in ccRCC. Mechanistically, Di-Ras2 exerts an activating effect on the MAPK pathway. Meanwhile, its activity is regulated by VHL, which can promote the ubiquitination and degradation of Di-Ras2. It's the first time to reveal the role of Di-Ras2 in ccRCC tumorigenesis and provide a new perspective of the relationship between VHL and the MAPK pathway in ccRCC.

\section{*Correspondence Author:}

Wei-Qiang Gao (gao.weiqiang@sjtu.edu.cn) or Li Li (lil@sjtu.edu.cn).

Address: Stem Cell Research Center, Ren Ji Hospital, School of Biomedical Engineering \& 
34 Med-X Research Institute, Shanghai Jiao Tong University, 160 Pujian Road, Shanghai, 200127, 35 China.

36 Tel: 86-158-2112-6637, Fax: 86-21-68383916.

37 
38

\section{Abstract}

Clear cell renal cell carcinoma (ccRCC) is one of the most common and lethal human urological malignancies in the world. The pathological drivers for ccRCC are still poorly understood. One of them is the Ras family of small GTPases that function as "molecular switches" in many diseases including ccRCC. Among them, Di-Ras2 encodes a 26-kDa GTPase that shares $60 \%$ homology to Ras and Rap. Yet little is known about the biological function(s) of Di-Ras2. In this study, we found that Di-Ras2 was upregulated in ccRCC, and promoted the proliferation, migration and invasion of human ccRCC cells in the absence of von Hippel-Lindau (VHL). Mechanistically, Di-Ras2 induces and regulates ccRCC formation by modulating phosphorylation of the downstream effectors and activating the MAPK signaling pathway. Moreover, Di-Ras2 interacts with E3 ubiquitin ligase, VHL, which facilitates the ubiquitination and degradation of Di-Ras2. Together, these results indicate a potential function of Di-Ras2 as an oncogene and biomarker in ccRCC, and these data provide a new perspective of the relationship between VHL and the MAPK pathway in ccRCC tumorigenesis. 


\section{Introduction}

Renal cell carcinoma (RCC) originates from the renal tubular epithelium and is the most common type of kidney cancer, accounts for $>90 \%$ of cancers in the kidney [1]. RCC is also the $7^{\text {th }}$ an $9^{\text {th }}$ most common cancer in men and women, respectively, worldwide, and accounts for approximately $2-3 \%$ of all adult malignancies [2-4]. Major subtypes with $\geq 5 \%$ incidence are clear cell RCC (ccRCC) [5], papillary RCC (pRCC) [] and chromophobe RCC (chRCC) [7]. The most common subtype of RCC is clear cell renal cell carcinoma (ccRCC), which accounts for $75-80 \%$ of all diagnosed cases [8]. Early diagnosis of the disease is difficult, and metastases often occur before the primary tumor is detected. Therefore, radical nephrectomy is considered to be the most effective way to treat ccRCC $[\underline{9}, \underline{10}]$. But the five-year survival rate for individuals with metastatic disease is still less than $10 \%$ [11]. It has been reported that hepatocyte growth factor (HGF) binding to its receptor, tyrosine-protein kinase Met, leads to activation of the Ras/MAPK signaling pathway to promote ccRCC growth and metastasis $[\underline{9}, \underline{12}]$. Function of the RAS-Mitogen activated protein kinase (MAPK) signaling pathway (also known as the RAS-RAF-MEK-ERK pathway) is to integrate extracellular signals and to coordinate a suitable response by a subsequent control of cellular growth, survival, and differentiation[13]. Aberrant activation of this pathway is a major and highly prevalent oncogenic event in many human cancers including ccRCC. Therefore, how Ras/MAPK signaling pathway is activated is important for us to understand the molecular mechanism of ccRCC, and suppression of Ras/MAPK signaling cascade may be one of the strategies to treat ccRCC.

The function of MAPK kinase is mediated by Ras GTPase signaling in normal and disease states $[\underline{14}, \underline{15}]$. As an important component in Ras/MAPK signaling pathway, the small GTPase 
Ras family regulates a wide range of cell functions, including proliferation, differentiation, migration and invasion[16, 17]. The GTP-binding Ras-like protein 2 (Di-Ras2) is one of GTPases forming a distinct subgroup of the Ras family, and shares 30-40\% overall identity with other members of the Ras family $[\underline{18}, \underline{19}]$. Di-Ras2 forms a tight complex with SmgGDS in cytosol, which lowers its affinity for guanine nucleotides [20]. Analyses using Oncomine and The Cancer Genome Atlas (TCGA) databases show that mRNA expression levels of Di-Ras2 are significantly up-regulated in some tumors, especially ccRCC. However, little is known about the biological role(s) of Di-Ras2 or how their activities are regulated in ccRCC.

ccRCC is genetically characterized by a frequent loss or mutation of von Hippel-Lindau (VHL) tumor suppressor gene, which appears to be the most important gene for the formation and progression of ccRCC $[1, \underline{5}, \underline{21]}$. The tumor-suppressive effect of VHL protein comes from its function as an E3 ubiquitin ligase complex substrate recognition element [22, 23] $]$.Moreover, VHL takes part in the ubiquitin-proteasome pathway (UPP) to control steady-state protein level of several effectors in ccRCC[24, 25]. Although Hypoxia-inducible factor $\alpha$-subunit (HIF) is the most studied gene interacting with VHL[26-28], recent studies also showed that VHL can interact with several other oncogenic proteins and facilitates their ubiquitination and degradation in ccRCC, such as Jade family PHD finger 1 (Jade-1)[29-31],Zinc fingers and homeoboxes 2 (ZHX2)[32], protein phosphatase 5 (PP5)[33], Aurora kinase A (AURKA)[34], and atypical protein kinase $\mathrm{C}(\mathrm{PKC})[35]$. Moreover, VHL has also been reported to regulate the epigenetic landscape of renal cancer by binding and degrading the hyperphosphorylated Rpb1 [36]. Therefore, according to its multifunction, von Hippel-Lindau (VHL) is highly related to ccRCC by enhancing the ubiquitination and degradation of certain oncogenes. However, whether and how VHL can 
97 directly interact with the MAPK pathway is unclear.

98 In the present study, we sought to characterize the physiological properties of Di-Ras2

99 proteins in ccRCC. To this end, we employed a plasmids-mediated overexpression and

100 knockdown system to determine Di-Ras2's function in ccRCC. We found that Di-Ras2 promoted

101 ccRCC proliferation by activating the MAPK pathway in the absence of von Hippel-Lindau

102 (VHL), which can facilitate the ubiquitination and degradation of Di-Ras2. We thus propose that

103 Di-Ras2 is a potential oncogene and biomarker in ccRCC, and these data provide us a new view of

104 the relationship of VHL and the MAPK pathway in ccRCC tumorigenesis. 


\section{Results}

\section{Di-Ras2 expression is up-regulated in ccRCC}

To determine the significance of Di-Ras2 in ccRCC, we first analyzed multiple microarray data sets in the Oncomine and TCGA databases. There were about 100 genes differentially expressed in human kidney tumor samples as compared to the adjacent normal kidney tissues. Among them, Di-Ras2 exhibited the most significant up-regulation in ccRCC samples (Supplementary Fig. S1). As shown in Figs. 1A-F, Di-Ras2 mRNA levels were increased significantly in human tumor samples as compared to the adjacent normal kidney tissues.

To verify the microarray analysis results, we performed q-PCR, Western blotting, and IHC staining on human ccRCC specimens and their matched normal tissues. Data showed that mRNA and protein expression levels of Di-Ras2 were significantly higher in renal cell carcinoma compared with normal tissues in most of the paired samples. (Figs. 1G-J).

\section{Di-Ras2 promotes VHL-mutated ccRCC tumor growth in vitro and in vivo.}

To examine the biological function of Di-Ras2 in ccRCC, we then employed lentivirus-mediated overexpression and knockdown systems in 786-O cells (Figs. 2A and 2B), which has been established as one of the first RCC cell lines with many characteristics of ccRCC, and is used most commonly in RCC-focused research [37]. As shown in Figs. 2C and 2D, cell proliferation was increased significantly by overexpression of Di-Ras2 and was suppressed by knockdown of Di-Ras2 in 786-O cells. In addition, results from cell wound scratch assays and matrigel invasion trans-well assays demonstrated that Di-Ras2 overexpression promoted cell migration and invasion abilities, and Di-Ras2 knockdown attenuated the migration and invasion abilities of 786-O cells 

promotes tumor progression in ccRCC cell lines in the absence of VHL. 
149 Given the fact that the Ras/MAPK pathways are often epigenetically dysregulated in ccRCC and

150 MAPK kinase cascade functions as an essential effector cascade required for Ras GTPase

151 signaling, we hypothesized that Di-Ras2may promote tumor growth through the MAPK pathway.

152 Indeed, in the absence of VHL, Di-Ras2 promoted the phosphorylation of several MAPK pathway

153 signal factors, such as MAPK, JNK, and P38 in 786-O cells. However, the MAPK pathway could

154 not be activated by Di-Ras2 in CAKI-1 cells (wild type VHL) (Fig. 4A). In addition, we

155 performed IHC and WB on xenograft tumor sections and samples as indicated in Fig. 3, and

156 obtained the same results (Figs. 4B and 4C). These data confirmed that Di-Ras2 can promote the

157 cascade reaction in the MAPK pathway in VHL-mutated ccRCC. To further validate the

158 suppressive role of VHL in the activation of the MAPK pathway induced by Di-Ras2, we

159 overexpressed VHL in 786-O cells and knocked down VHL in CAKI-1 cells. As shown in Fig. 4D,

160 Di-Ras2 activated the MAPK signaling pathway in VHL-mutated 786-O cells, while this function

161 was blocked by exogenous VHL. Meanwhile, the activation of MAPK signaling pathway was

restored after knockdown of VHL in Di-Ras2-transfected CAKI-1 cells (wild type VHL). declined (Figs. 4D and 4E). 
VHL interacts with Di-Ras2 and enhances the ubiquitination and degradation of Di-Ras2

173 Increasing evidence indicates that the E3 ligase plays an important role in oncogenesis and cancer

174 development by controlling protein levels that regulate multiple biological processes [38]. Given

175 that VHL is a type of E3 ligase, we next investigated the molecular mechanism through which

176 VHL promoted Di-Ras2 degradation. As shown in Fig. 5A, the half-life of Di-Ras2 protein was

177 shortened significantly in 293T and CAKI-1 cells transfected with VHL after using cycloheximide

178 (CHX), indicating that VHL negatively affects Di-Ras2 stability. In addition, as shown in Fig. 5B,

179 we observed that treatment with MG132, a proteasome inhibitor, led to a significant lengthening of the Di-Ras2 protein half-life, supporting the notion that VHL regulates Di-Ras2 stability by proteasome-mediated mechanisms. next explored whether VHL interacts with Di-Ras2. Co-immunoprecipitation results showed that both exogenous and endogenous VHL bound to Di-Ras2 in 293T cells and CAKI-1 cells (Figs. 5C and 5D). Considering that VHL protein serves as an E3 ligase, we hypothesized that VHL promoted Di-Ras2 protein ubiquitination. In agreement with our hypothesis, we observed that the ubiquitination of both exogenous and endogenous Di-Ras2 was augmented by VHL overexpression, whereas they were diminished by VHL knockdown in 293T cells and CAKI-1 cells respectively (Fig. 5E). To confirm the interaction between VHL and Di-Ras2, we employed its ubiquitination and degradation. 
The tumor-promoting activity of Di-Ras2 is overridden by VHL.

195 To verify the suppressive role of VHL in Di-Ras2-induced ccRCC progression, we then employed

a lentivirus-mediated overexpression system of VHL on Di-Ras2 overexpressed 786-O

Meanwhile, cell proliferation was increased by knockdown of VHL in Di-Ras2-OV CAKI-1 cells

CAKI-1 cells (Fig. 6D). We also performed xenograft tumor assays using these cell lines.

VHL and Di-Ras2 promotes ccRCC tumor progression in the absence of VHL.

211 The results showed that the ubiquitination of Di-Ras2 was augmented by VHL overexpression in 
215 Given our observations that Di-Ras2 can upregulate the MAPK pathway in human ccRCC and 216 that VHL negatively regulated Di-Ras2 protein stability, we re-analyzed the TCGA RPPA data and 217 found that the phosphorylation level of p38MAPK was significantly up-regulated in VHL-mut and 218 DIRAS2-high ccRCC samples, which implicates an activation of the MAPK pathway in these 219 samples (Fig. 7A). Further analyses showed a weak inverse correlation between the mRNA levels 220 of Di-Ras2 and that of VHL in ccRCC samples from TCGA database (Fig. 7B). We also compared 221 the Di-Ras2 and VHL protein levels from surgically removed human kidney tumor samples and 222 RCC cell lines, using western blots and immunohistochemical staining, and found a clear inverse correlation between Di-Ras2 and VHL protein levels (Figs. 7C-E), which indicated that

224 protein-protein interaction should play a dominant role in the downregulation of Di-Ras2. These 225 findings suggested that the deficiency of VHL in ccRCC appear to promote the stability of 226 Di-Ras2, leading to MAPK pathway activation, resulting in an accelerated tumor growth and progression (Fig. 7F). 


\section{Discussion} regulation of the protein stability of HIFs is the most recognizable activity of VHL $[\underline{35}, \underline{36}, \underline{45}]$.

The small GTPase Ras family proteins are appreciated as potential candidates in many cancers.

For example, the Ras/MAPK signaling pathway is altered in pancreatic ductal adenocarcinoma (PDAC), melanoma, lymphoblastic leukemia, lung adenocarcinomas, colon and rectal cancer including mutations in NRAS, KRAS or BRAF [39-44]. Moreover, the current study reinforces the idea that Ras/MAPK signaling pathway is activated predominantly with ccRCC $[\underline{9}, \underline{12}]$. For patients with surgically resectable ccRCC, the standard of care is surgical excision by either partial or radical nephrectomy with a curative intent. By contrast, those with inoperable ccRCC typically undergo systemic treatment with targeted agents and/or immune checkpoint inhibitors $[\underline{1}, \underline{10}]$. Accordingly, Ras/MAPK signaling pathway could be a promising target for ccRCC treatment. As a member of the Ras subfamily, yet little is known about the biological function(s) of Di-Ras2 in cancer research. Based on the MAPK signaling activation and its association with VHL, we have uncovered a specific function of Di-Ras2 in ccRCC as an activator of MAPK signaling pathway in the absence of VHL, which can facilitate the ubiquitination and degradation of Di-Ras2 and block its function in ccRCC. Our results thus implicate that Di-Ras2 is a novel oncogene which contributes to the formation of ccRCC and a potential target of VHL, linking the kidney-specific VHL tumor suppressor pathway and the MAPK signaling cascade in ccRCC.

VHL is a multifunctional protein and has been closely associated with ccRCC. To date, the most well-characterized function of VHL is its role as an E3 ubiquitin ligase that participates in the degradation of some oncogenic effectors. Of the several targets mentioned above, the 
251 enriched in ccRCC cells in which VHL is lost or mutated $[\underline{32}, \underline{46]}$. We showed in current study a 252 potential target of VHL, Di-Ras2, and revealed a role of VHL in the regulation of ccRCC 253 formation through the Ras/MAPK pathway. VHL physically binds to Di-Ras2 and enhances its 254 ubiquitination and degradation. The loss of VHL results in dramatic changes in regulation of 255 Di-Ras2, which can activate the MAPK pathway and induce ccRCC. This observation for the first 256 time demonstrates that VHL can inhibit the MAPK signaling pathway through interaction with 257 Di-Ras2. Taken together, our findings have demonstrated the biological and clinical significance of Di-Ras2 as a potential oncogene in ccRCC. Di-Ras2 exerts an activating effect on the MAPK pathway and its downstream signals, thereby promoting ccRCC cells proliferation, migration and

261 invasion. Meanwhile, its activity is regulated by VHL, which can promote the ubiquitination and degradation of Di-Ras2. In addition, our data suggest that activation of the MAPK pathway by Di-Ras2 represents a novel tumor promoter axis for VHL/Di-Ras2/MAPK. Furthermore, these

264 findings directly link the kidney-specific VHL tumor suppressor pathway and the MAPK signaling 265 cascade, and enlarge the scope of basic research on ccRCC formation. For clinical translation, 266 pharmaceutical investigation of the interaction between Di-Ras2 and VHL may provide a potential promising strategy to inactivate MAPK signaling in ccRCC. 


\section{Materials and Methods}

271

272

273

274

\section{Patient samples}

Microarray data from the Oncomine and TCGA databases were used. Twenty fresh samples of human ccRCC and paired normal tissue were obtained during surgery at the Department of General Surgery, Ren Ji Hospital. All samples were collected with the informed consent of patients and approved by the Animal Care committee of Shanghai Ren Ji Hospital. All cDNA and protein samples were extracted from those fresh tissue samples.

\section{RNA isolation and quantitative RT-PCR}

Total RNA was isolated from cultured cells or fresh samples with Trizol reagent (Invitrogen). cDNA was synthesized by reverse transcription using the Prime Script RT reagent kit (TaKaRa) and subjected to quantitative RT-PCR with Di-Ras2, VHL, and ACTB or GAPDH primers in the presence of the SYBR Green Realtime PCR Master Mix (Thermo). Relative abundance of mRNA was calculated by normalization to ACTB or GAPDH mRNA. Data were analyzed from three independent experiments and were shown as the mean \pm SEM.

\section{Immunoblotting}

Cells were lysed with $100-300 \mu \mathrm{L}$ RIPA buffer supplemented with protease and phosphatase inhibitors (Millipore). The protein concentration was measured with the BCA Protein Assay (Bio-Rad). From each sample, $20-50 \mu \mathrm{g}$ of total protein was separated by $8-12 \%$ SDS-PAGE gels and transferred onto nitrocellulose membranes (GM). Membranes were blocked in 5\% BSA in TBS for 1 hour at room temperature, and then incubated with primary antibodies overnight at $4{ }^{\circ} \mathrm{C}$, 
washed in TBS containing $1 \%$ Tween20, incubated with an HRP-conjugated secondary antibody for 1 hour at room temperature, and developed by ECL reagent (Thermo). The immunoblots were quantified by Bio-Rad Quantity One version 4.1 software. Primary antibodies against Di-Ras2 (Proteintech ag7926), VHL (SAB \#32075), and ubiquitin (sc-8017) were purchased from

\section{Immunofluorescent and immunohistochemistry (IHC) assays}

311 For IHC staining, paraffin-embedded ccRCC tissues were deparaffinized, rehydrated, and 
314 The sections were then incubated with $0.3 \%$ Triton X-100 in PBS (137 mM NaCl, $2.7 \mathrm{mM} \mathrm{KCl}$,

$31510 \mathrm{mM} \mathrm{Na}_{2} \mathrm{HPO}_{4}, 2 \mathrm{mM} \mathrm{KH}_{2} \mathrm{PO}_{4}, \mathrm{pH}$ 7.4) for 15 minutes, followed by $10 \%$ goat serum in PBS

316 for 1 hour. Subsequently, samples were incubated with a mouse polyclonal Di-Ras2 antibody

317 (Abcam, ab67430), diluted at 1:500 in $1 \%$ goat serum for 1 hour at $37^{\circ} \mathrm{C}$. After three washes in

318 PBS, sections were incubated with an HRP-conjugated secondary antibody for 1 hour at room temperature. Sections were counterstained with hematoxylin. Three random immunostaining

320 images of each specimen were captured using a Leica DM2500 microscope and analyzed by 321 Image-Pro Plus 6.0 software.

\section{Immunoprecipitation and ubiquitination assays}

324 Cells were washed with ice-cold PBS and lysed in RIPA lysis buffer $(50 \mathrm{mmol} / \mathrm{L}$ Tris-HCl, $\mathrm{pH}$ 8.0; $150 \mathrm{mmol} / \mathrm{L} \mathrm{NaCl} ; 1 \% \mathrm{NP}-40$ ) supplemented with protease and phosphatase inhibitors (Millipore) at 24 hours after transfection. Cell lysates were incubated with primary antibodies overnight at $4^{\circ} \mathrm{C}$. Pierce $^{\mathrm{TM}}$ Protein A/G Magnetic Beads (Thermo) were then added and the lysates were incubated

328 for another 4 hours at $4^{\circ} \mathrm{C}$. The immunoprecipitates were washed 4 times with the lysis buffer and 329 boiled for 5 minutes at $98^{\circ} \mathrm{C}$ in protein loading buffer. Immunoprecipitated proteins were detected 330 by subsequent immunoblotting. Antibodies used in the co-immunoprecipitation experiments were 331 as follows: anti-Di-Ras2 (Proteintech ag7926), anti-VHL (SAB \#32075), anti-Myc-tag(CST 332 \#2276), anti-HA(CST \#5017), mouse immunoglobulin G, rabbit immunoglobulin G (Santa Cruz), 333 glyceraldehyde-3-phosphate dehydrogenase (CST), and anti-ubiquitin (Santa Cruz sc-7199) antibodies. 


\section{Protein half-life detection}

HEK293T and CAKI-1 cells were co-transfected with the Di-Ras2-Myc expression plasmid and

338 the VHL-Flag expression plasmid or the empty vector as described earlier. A total of $10 \mu \mathrm{g} / \mathrm{mL}$ of

339 cycloheximide (CHX, purchased from Sigma) was added to the culture medium 24 hours after the

340 plasmid transfection to 293T cells and CAKI-1 cells. Cells were lysed in RIPA buffer containing

341 protease and phosphatase inhibitors as described earlier, after CHX treatment at indicated time

342 points. For MG132 treatment, at indicated hours after transfection, cells were incubated with

343 MG132 $(10 \mu \mathrm{mol} / \mathrm{L})$ for an additional 6 or 12 hours. Cells were then collected for immunoblots to

344 determine the amount of Di-Ras2 protein.

346 Cell lines and culture

347 All cell lines were obtained from the American Type Culture Collection (ATCC) and were

348 confirmed by specific indices. CAKI-1, 786-O, A498, and 293T were cultured in RPMI-1640 or

349 DMEM supplemented with $10 \%$ FBS (Thermo), $100 \mathrm{U} / \mathrm{mL}$ penicillin, and $0.1 \mathrm{mg} / \mathrm{mL}$

350 streptomycin (Thermo) at $37^{\circ} \mathrm{C}$ in humidified $5 \% \mathrm{CO}_{2}$ atmosphere. Hypoxic conditions were

351 achieved with a hypoxia chamber (Billups-Rothenberg) flushed with a gas mixture of $1 \% \mathrm{O} 2,5 \%$

$352 \mathrm{CO} 2$ and $94 \% \mathrm{~N} 2$.

355 ShRNA sequences for VHL and scramble shRNA were cloned into lentiviral vector 
Human Di-Ras2 and VHL cDNA was generated by polymerase chain reaction and cloned into

VHL were cloned into the lentiviral expression vector pLenti.CMV.Puro.DEST. generate the stable transfections.

\section{Cell proliferation assays}

372 Cells were plated in 96-well plates and examined at 24, 48, 72, and 96 hours after plating $(n=3)$.

373 Cells were incubated with CellTiter 96 AQueous (MTS) solution for 3 hours. The absorbance at

$374490 \mathrm{~nm}$ was then measured using a microplate reader (BioTek).

\section{Cell scratch wound healing assay}

377 Cells were plated at a density of $1 \times 10^{5}$ cells/well in triplicate into 6-well plates. Once the cells had spread over the bottom of the wells, three or four parallel lines were scratched into each well using sterile $10 \mu \mathrm{L}$ tips. Suspended cells were washed off and remaining cells were cultured in medium 
without FBS. After 12 and 24 hours of incubation, for each well, five random fields were examined under a light microscope, photographed, and counted manually.

\section{Migration assay}

Costar Trans-well migration plates with $8 \mu \mathrm{m}$ pore size (Corning, \#3422) were pre-coated with

Matrigel. Cells $\left(1 \times 10^{5}\right)$ in $100 \mu$ LRPMI medium without FBS were placed in triplicate into the upper chamber. To the lower chamber, $500 \mu \mathrm{L}$ medium containing 10\% FBS was added. After 12 and 24 hours of incubation, the plate inserts were removed and washed with PBS buffer several times to get rid of unattached cells. All the residual cells on the upper side were scraped with a cotton swab. Migrated cells on the lower side of the insert were fixed in $4 \%$ formalin for 20 minutes, washed twice with PBS, and stained with $0.1 \%$ crystal violet for 10 minutes. For each insert, five random fields were examined under a light microscope, photographed, and counted manually.

\section{In vivo xenograft assay}

395 Cell suspensions $\left(1 \times 10^{6}\right.$ cells $)$ of cells, in a total volume of $100 \mu \mathrm{L}$ mixed with Matrigel in a 1:1 ratio, were injected subcutaneously into the right flanks of 4-week-old male BALB/C nude mice

397 (SLAC, Shanghai). The body weight and tumor volumes were measured and recorded every 3

398 days from 2 weeks after inoculation. Tumor volume was calculated with the following formula:

399 volume $=0.5 \times$ tumor length $\times$ tumor width $^{2}$. Tumors were collected and photographed 60 days 400 after inoculation. All mice were housed in the SPF animal facility in a pathogen-free environment 401 with controlled temperature and humidity. All animal experiments were carried out following the 
402 ethical regulations of the Animal Care committee at Ren Ji Hospital.

403

\section{$404 \quad$ Statistical analysis}

405 Statistical evaluation was conducted using Student's t-test. Multiple comparisons were analyzed 406 first by one-way analysis of variance. The log-rank (Mantel-Cox) test was used for patient survival analysis. The Pearson correlation was used to analyze the strength of the association between expression levels of Di-Ras2 and its related genes in patient samples. A significant difference was defined as $\mathrm{P}<0.05$.

Data availability statement

412 The data that support the findings of this study are available from the corresponding author upon reasonable request.

\section{Acknowledgements}

416 This study was supported by funds from Ministry of Science and Technology of the People's 417 Republic of China (2017YFA0102900 to WQG), National Natural Science Foundation of China 418 (81872406 and 81630073 to WQG, 81772938 to L. Li, 81602443 to X. Li), State Key Laboratory 419 of Oncogenes and Related Genes (KF01801 to L. Li), Science and Technology Commission of 420 Shanghai Municipality (16JC1405700 to WQG, 18140902700 and 19140905500 to L. Li), High 421 Peak IV fund from Education Commission of Shanghai Municipality on Stem Cell Research (to 
424 Municipal Education Commission (ZXGF082101), and Shanghai Jiao Tong University Medical

425 Engineering Cross Fund (YG2016MS52).

426

\section{Author contributions}

428 HR mainly performed the experiments, analyzed the data and wrote the paper. XL collected the

429 clinical samples. ML, JL, XL and JX helped with the experiments. LL and W-Q Gao carried out

430 the experiment design and manuscript drafting. All authors had edit and approved the final

431 manuscript.

432

\section{Conflict of interest}

434 The authors declare that they have no competing interests exist.

435 
436

437

438

439

440

441

442

444

445

446

\section{References}

1. Hsieh, J.J., et al., Renal cell carcinoma. Nat Rev Dis Primers, 2017. 3: p. 17009.

2. Rini, B.I., S.C. Campbell, and B. Escudier, Renal cell carcinoma. The Lancet, 2009. 373(9669): p. 1119-1132.

3. Ferlay, J., et al., Cancer incidence and mortality worldwide: sources, methods and major patterns in GLOBOCAN 2012. Int J Cancer, 2015. 136(5): p. E359-86.

4. Siegel, R.L., K.D. Miller, and A. Jemal, Cancer Statistics, 2017. CA Cancer J Clin, 2017. 67(1): p. 7-30.

5. Cancer Genome Atlas Research, N., Comprehensive molecular characterization of clear cell renal cell carcinoma. Nature, 2013. 499(7456): p. 43-9.

6. Cancer Genome Atlas Research, N., et al., Comprehensive Molecular Characterization of Papillary Renal-Cell Carcinoma. N Engl J Med, 2016. 374(2): p. $135-45$.

7. Davis, C.F., et al., The somatic genomic landscape of chromophobe renal cell carcinoma. Cancer Cell, 2014. 26(3): p. 319-330.

8. Moch, H., et al., The 2016 WHO Classification of Tumours of the Urinary System and Male Genital Organs-Part A: Renal, Penile, and Testicular Tumours. Eur Urol, 2016. 70(1): p. 93-105.

9. Morris, M.R. and F. Latif, The epigenetic landscape of renal cancer. Nat Rev Nephrol, 2017. 13(1): p. 47-60.

10. Alt, A.L., et al., Survival after complete surgical resection of multiple metastases from renal cell carcinoma. Cancer, 2011. 117(13): p. 2873-82. 
458 11. Stadler, W.M., Targeted agents for the treatment of advanced renal cell carcinoma. Cancer, 2005. 104(11): p. 2323-33.

12. Banumathy, G. and P. Cairns, Signaling pathways in renal cell carcinoma. Cancer Biol Ther, 2010. 10(7): p. 658-64.

13. J, D., Targeting RAS signalling pathways in cancer therapy. Nat Rev Cancer, 2003. 3(1): p. 11-22.

14. Aoki, Y., et al., The RAS/MAPK syndromes: novel roles of the RAS pathway in human genetic disorders. Hum Mutat, 2008. 29(8): p. 992-1006.

15. Tidyman, W.E. and K.A. Rauen, The RASopathies: developmental syndromes of Ras/MAPK pathway dysregulation. Curr Opin Genet Dev, 2009. 19(3): p. 230-6.

16. Foster, S.A. and S. Malek, The RAS/MAPK Axis Gets Stressed Out. Mol Cell, 2016. 64(5): p. 854-855.

17. Ritt, D.A., et al., Inhibition of Ras/Raf/MEK/ERK Pathway Signaling by a

472 18. Kontani, K., et al., Di-Ras, a distinct subgroup of ras family GTPases with unique biochemical properties. J Biol Chem, 2002. 277(43): p. 41070-8.

474 19. Gasper, R., B. Sot, and A. Wittinghofer, GTPase activity of Di-Ras proteins is stimulated by Rap1GAP proteins. Small GTPases, 2010. 1(3): p. 133-141.

476 20. Ogita, Y., et al., Di-Ras2 Protein Forms a Complex with SmgGDS Protein in Brain Cytosol in Order to Be in a Low Affinity State for Guanine Nucleotides. J Biol Chem, 2015. 290(33): p. 20245-56.

479 21. Tang, X., et al., Cystine Deprivation Triggers Programmed Necrosis in VHL-Deficient 
Renal Cell Carcinomas. Cancer Res, 2016. 76(7): p. 1892-903.

481

22. Hon WC1, W.M., Harlos K, Claridge TD, Schofield CJ, Pugh CW, Maxwell PH, Ratcliffe PJ, Stuart DI, Jones EY., Structural basis for the recognition of hydroxyproline in HIF-1 alpha by pVHL. Nature 2002. 417(27): p. 975-978.

23. Maniaci, C., et al., Homo-PROTACs: bivalent small-molecule dimerizers of the VHL E3 ubiquitin ligase to induce self-degradation. Nat Commun, 2017. 8(1): p. 830.

24. Tu1., Y., et al., The Ubiquitin Proteasome Pathway (UPP) in the regulation of cell cycle control and DNA damage repair and its implication in tumorigenesis.pdf $>$. Int $\mathrm{J}$ Clin Pathol, 2012. 5(8): p. 726-738.

25. Brinkmann, K., et al., Regulation of the DNA damage response by ubiquitin conjugation. Front Genet, 2015. 6: p. 98.

26. Robinson, C.M., et al., Consequences of VHL Loss on Global DNA Methylome. Sci Rep, 2018. 8(1): p. 3313.

27. Chittiboina, P. and R.R. Lonser, Von Hippel-Lindau disease. Handb Clin Neurol, 2015. 132: p. 139-56.

28. Li, Q., et al., E3 Ligase VHL Promotes Group 2 Innate Lymphoid Cell Maturation and Function via Glycolysis Inhibition and Induction of Interleukin-33 Receptor. Immunity, 2018. 48(2): p. 258-270 e5.

29. Chitalia, V.C., et al., Jade-1 inhibits Wnt signalling by ubiquitylating beta-catenin and mediates Wnt pathway inhibition by pVHL. Nat Cell Biol, 2008. 10(10): p. 1208-16.

30. Xiao-Fen, W., et al., Correlation analysis of VHL and Jade-1 gene expression in human renal cell carcinoma. Open Med (Wars), 2016. 11(1): p. 226-230. 
502 31. Zeng, L., et al., Candidate tumor suppressor and pVHL partner Jade-1 binds and

503

504

505

506

507

508

509

510

511

512

513

514

515 inhibits AKT in renal cell carcinoma. Cancer Res, 2013. 73(17): p. 5371-80.

32. Zhang., J., et al., VHL substrate transcription factor ZHX2 as an oncogenic driver in clear cell renal carcinoma. Science, 2018. 361: p. 290-295.

33. Dushukyan, N., et al., Phosphorylation and Ubiquitination Regulate Protein Phosphatase 5 Activity and Its Prosurvival Role in Kidney Cancer. Cell Rep, 2017. 21(7): p. 1883-1895.

34. Hasanov, E., et al., Ubiquitination and regulation of AURKA identifies a hypoxia-independent E3 ligase activity of VHL. Oncogene, 2017. 36(24): p. 3450-3463.

35. Okuda, H., et al., The von Hippel-Lindau tumor suppressor protein mediates ubiquitination of activated atypical protein kinase C. J Biol Chem, 2001. 276(47): p. $43611-7$

36. Kuznetsova., A.V., et al., von Hippel-Lindau protein binds hyperphosphorylated large subunit of RNA polymerase II through a proline hydroxylation motif and targets it for ubiquitination. Proceedings of the National Academy of Sciences of the United States of America, 2003. 100(5): p. 2706-2711

37. Brodaczewska, K.K., et al., Choosing the right cell line for renal cell cancer research. Mol Cancer, 2016. 15(1): p. 83.

38. Yang, L., et al., Novel Insights Into E3 Ubiquitin Ligase in Cancer Chemoresistance. Am J Med Sci, 2018. 355(4): p. 368-376.

39. Biankin, A.V., et al., Pancreatic cancer genomes reveal aberrations in axon guidance 
pathway genes. Nature, 2012. 491(7424): p. 399-405.

40. Cancer Genome Atlas, N., Comprehensive molecular characterization of human colon and rectal cancer. Nature, 2012. 487(7407): p. 330-7.

41. Hodis, E., et al., A landscape of driver mutations in melanoma. Cell, 2012. 150(2): p. 251-63.

42. Imielinski, M., et al., Mapping the hallmarks of lung adenocarcinoma with massively parallel sequencing. Cell, 2012. 150(6): p. 1107-20.

43. Paik, P.K., et al., Clinical characteristics of patients with lung adenocarcinomas harboring BRAF mutations. J Clin Oncol, 2011. 29(15): p. 2046-51.

44. Zhang, J., et al., The genetic basis of early T-cell precursor acute lymphoblastic leukaemia. Nature, 2012. 481(7380): p. 157-63.

45. Schermer, B., et al., The von Hippel-Lindau tumor suppressor protein controls ciliogenesis by orienting microtubule growth. J Cell Biol, 2006. 175(4): p. 547-54. autocrine tumor-promoting network in epithelial ovarian cancer cells. Cancer Res, 


\section{Figure Legends}

Figure 1. Di-Ras2 expression is up-regulated in ccRCC. (A-F) Analysis from the Oncomine

543 and TCGA databases showed that mRNA expression levels of Di-Ras2 were significantly higher

544 in renal cell carcinoma compared with normal tissues. (G-H) Di-Ras2 mRNA expression level in

54514 paired tumor samples and normal tissue. (I) Immunoblotting showed higher protein levels of

546 Di-Ras2 in 5 of 6 tumor samples compared with the respective matched normal tissues (T, tumor;

$547 \mathrm{~N}$, normal tissue). (J) Immunohistochemical staining on normal and renal cell carcinoma tissues

548 with anti-Di-Ras2 antibody.

Figure 2. Di-Ras2 promotes ccRCC cell proliferation, migration, and invasion in

551 VHL-mutated cell lines. mRNA and protein levels of Di-Ras2 after (A) overexpression and (B)

552 knockdown of Di-Ras2 in 786-O cells lines. (C, D) Cell proliferation abilities were compared with

553 their vector control cells. Cell migration and invasion abilities of (E) DIRAS2-OV 786-O cells as

554 well as (F) DIRAS2-KD 786-O cells were compared with their vector control cells, as 555 demonstrated by cell wound scratch and trans-well assays. The percentages of (G) DIRAS2-OV

556 786-O cells as well as (H) DIRAS2-KD 786-O cells in the G1, S, and G2 phases for each sample 557 are shown. VT, vector; WT, wild-type; MUT, mutated; OV, overexpression; KD, knockdown. Data 558 are shown as the mean value \pm SEM.

560 Figure 3. Di-Ras2 promotes VHL-mutated ccRCC tumor growth in vivo. (A) Xenograft tumor 561 assays using 786-O cells stably transfected by DIRAS2-overexpression and sh-DIRAS2 562 lentiviruses. (B) Tumor volumes of xenografts generated by DIRAS2-OV and DIRAS2-KD 786-O 
563 cells were compared with their vector control cells. (C) Tumor weights of xenografts generated by

564 DIRAS2-OV and DIRAS2-KD 786-O cells were compared with their vector control cells.

565 Xenograft tumor assays using CAKI-1 cells stably transfected by DIRAS2-overexpression

566 lentiviruses. (E) Tumor volumes of xenografts generated by DIRAS2-OV CAKI-1 cells were 567 compared with their vector control cells. (F) Tumor weights of xenografts generated by 568 DIRAS2-OV CAKI-1 cells were compared with their vector control cells. Data are shown as the 569 mean value \pm SEM.

Figure 4. Di-Ras2 activates the MAPK pathway in the absence of VHL. (A) Overexpression of Di-Ras2 activated the MAPK pathway in VHL-mut 786-O cells, but not VHL-wt CAKI-1 cells. (B) IHC on xenograft tumor sections. (C) WB on xenograft tumor samples. (D) VHL attenuated the function of Di-Ras2 in activating MAPK in 786-O cells, and the deficiency of VHL restored the function of Di-Ras2 in activating MAPK in CAKI-1 cells. (E) The protein level of endogenous Di-Ras2 after overexpression and knockdown of VHL in $293 \mathrm{~T}$ and CAKI-1 cells (48h). (F) The

577 protein level of Di-Ras2 decreased in tandem with the progressively increasing levels of VHL in $578293 \mathrm{~T}$ and CAKI-1 cells (24h). (G) The expression of VHL reduced the protein abundance of co-transfected Di-Ras2 in 293T cells and CAKI-1 cells. 
CAKI-1 cells transfected with Myc-tagged Di-Ras2 and HA-tagged VHL. (D) Endogenous VHL with anti-Di-Ras2 and anti-VHL antibodies. and tans-well assays. (E) Xenograft tumor assays using DIRAS2-OV 786-O cells stably

(G) Xenograft tumor assays using DIRAS2-OV CAKI-1 cells stably transfected by sh-VHL 
Figure S2. Di-Ras2 has no effect on VHL-wild-type CAKI-1 cells. mRNA and protein levels of 
VHL-mutated A498 cells. mRNA and protein levels of Di-Ras2 after (A) overexpression and (B)

630

631

634

635

636

637

638

639

640

641

642

643

644

645

646

647

knockdown of Di-Ras2 in A498 cells lines. (C, D) Cell proliferation abilities were compared with their vector control cells. Cell migration and invasion abilities of (E) DIRAS2-OV A498 cells as well as (F) DIRAS2-KD A498 cells were compared with their vector control cells, as demonstrated by cell wound scratch and trans-well assays. The percentages of (G) DIRAS2-OV A498 cells as well as (H) DIRAS2-KD A498 cells in the G1, S, and G2 phases for each sample are shown. VT, vector; WT, wild-type; MUT, mutated; OV, overexpression; KD, knockdown. Data are shown as the mean value \pm SEM.

Figure S4. Di-Ras2 has no effect on VHL-wild-type ACHN cells. mRNA and protein levels of Di-Ras2 after overexpression (A) and knockdown (B) of Di-Ras2 in ACHN cells. (C, D) Cell proliferation abilities were compared with their vector control cells. VT, vector; WT, wild-type cells; MUT, VHL-mut cell; OV, overexpression Di-Ras2 lentivirus-infected cells; KD, shRNA lentivirus-infected cells. Data are shown as the mean value \pm SEM.

Figure S5. VHL mediated ubiquitination of Di-Ras2 is independent of hypoxia. (A) The protein abundance of Di-Ras2 in 786-O cells under hypoxia culture. (B) Ubiquitination of exogenous Di-Ras2 were enhanced by VHL overexpression in 293T cells and CAKI-1 cells under both normal and hypoxia culture. 
A

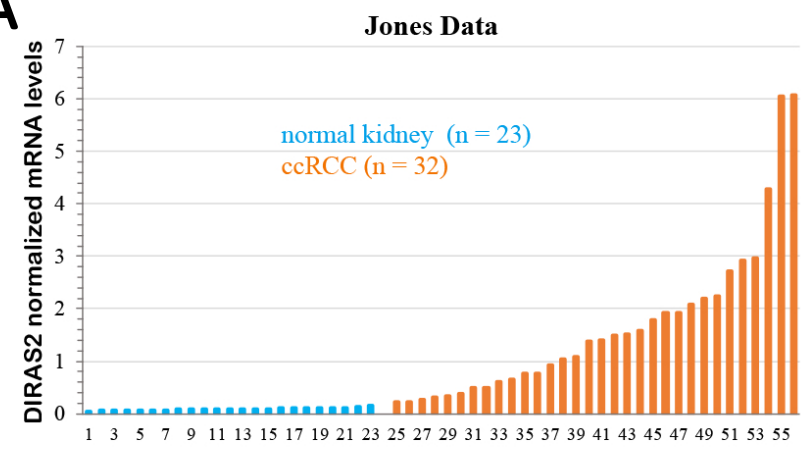

C
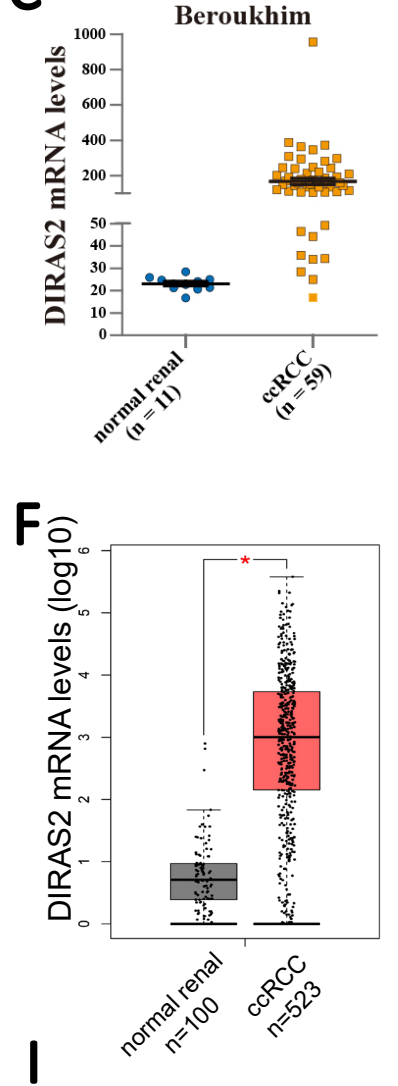

B

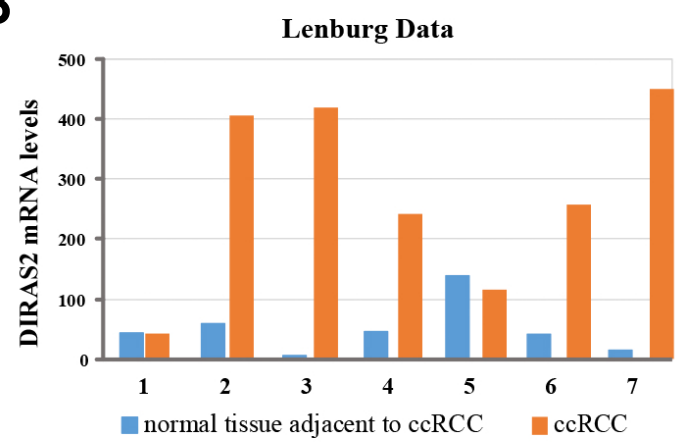

E

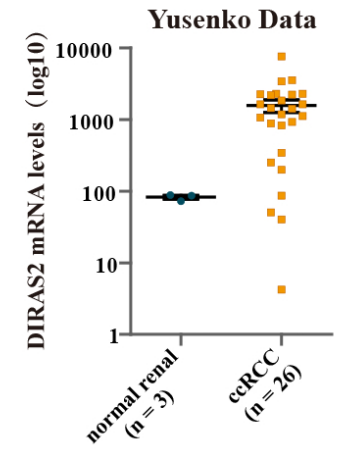

$\mathrm{H}$

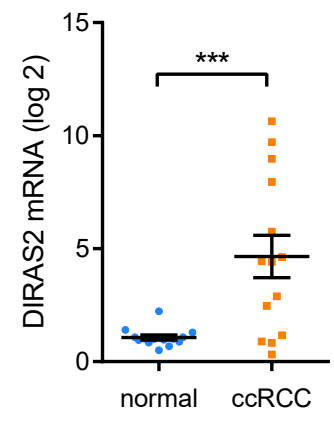

J

Patient 1

cCRCC

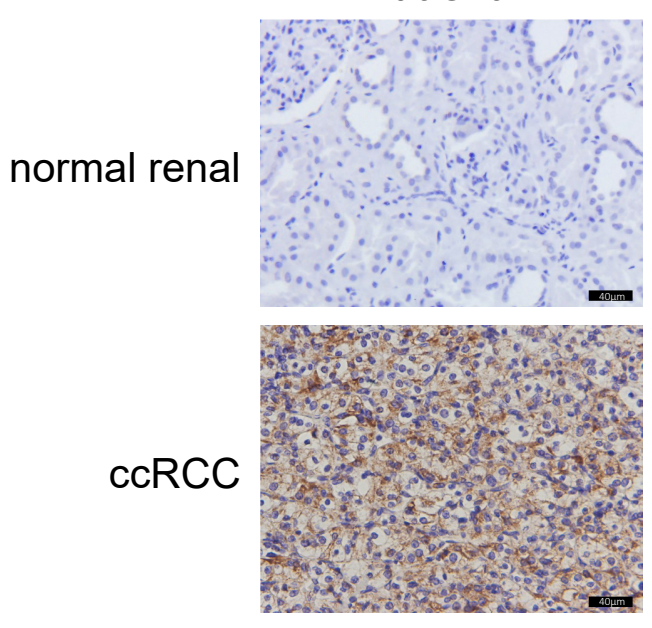

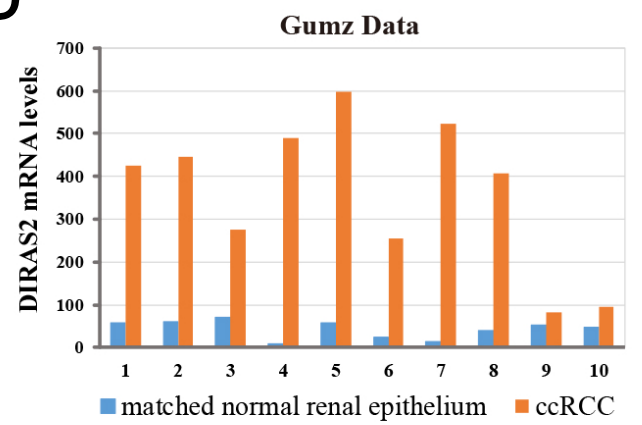

$\mathbf{G}$

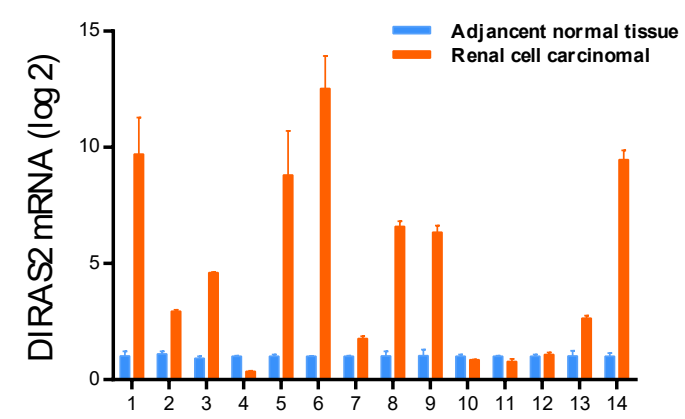

$\begin{array}{llllllllllll}\mathrm{N} 1 & \mathrm{~T} 1 & \mathrm{~N} 2 & \mathrm{~T} 2 & \mathrm{~N} 3 & \mathrm{~T} 3 & \mathrm{~N} 4 & \mathrm{~T} 4 & \mathrm{~N} 5 & \mathrm{~T} 5 & \mathrm{~N} 6 & \mathrm{~T} 6\end{array}$

Di-Ras2

GAPDH

normal renal

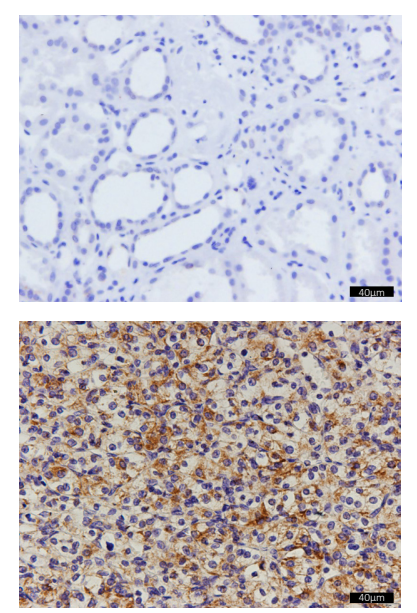

Patient 3

Patient 2

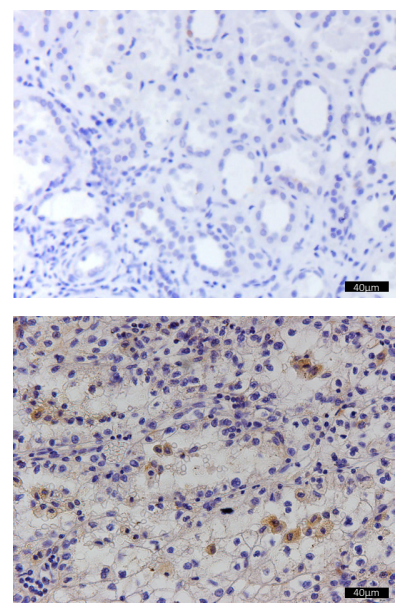


Figure 2

A

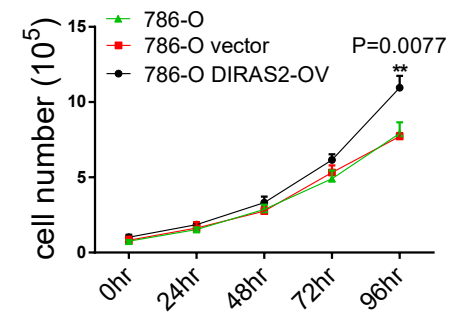

$\mathrm{E}$
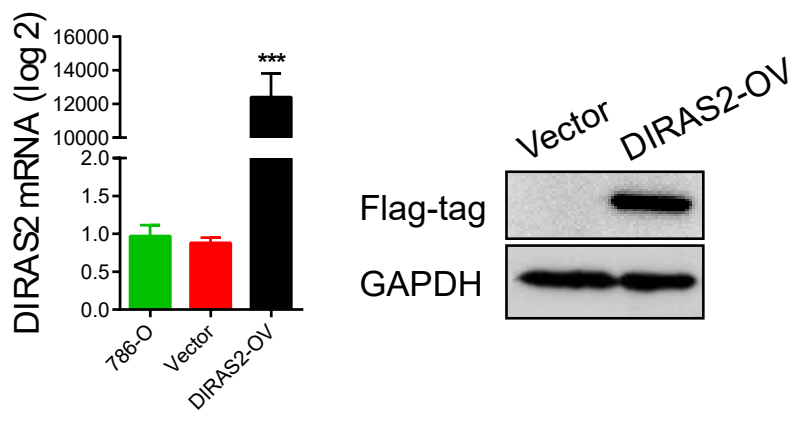

786-O cells (VHL-mut)
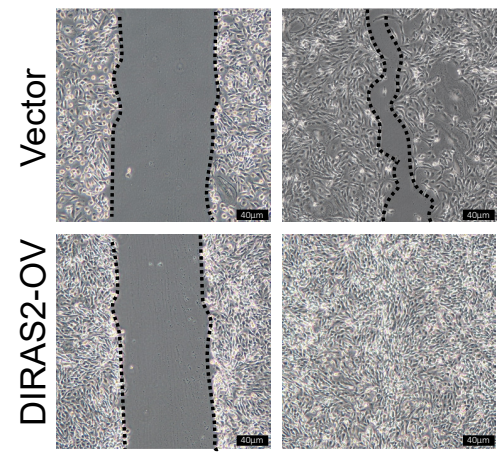

$12 \mathrm{~h}$

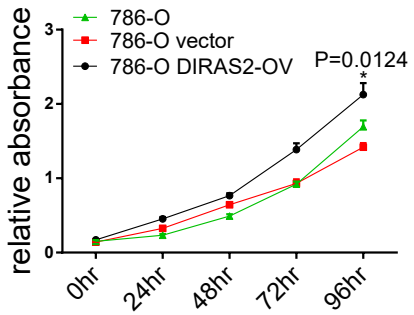

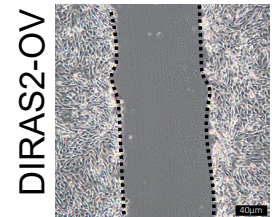

$\mathrm{Oh}$
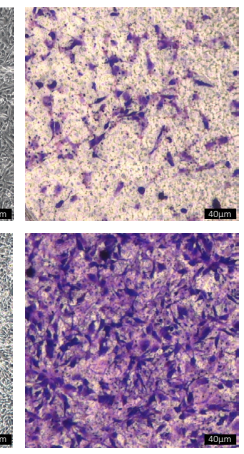

$12 \mathrm{~h}$

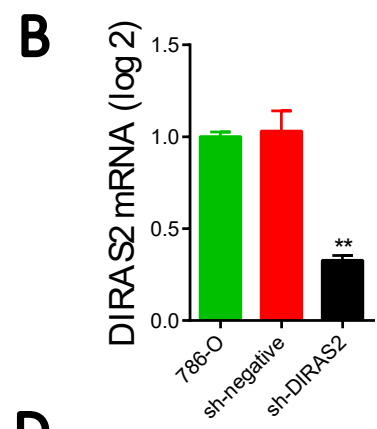

D
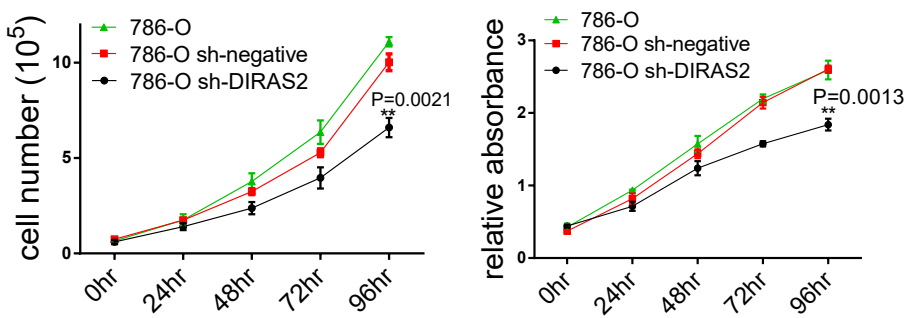

F 786-O cells (VHL-mut)
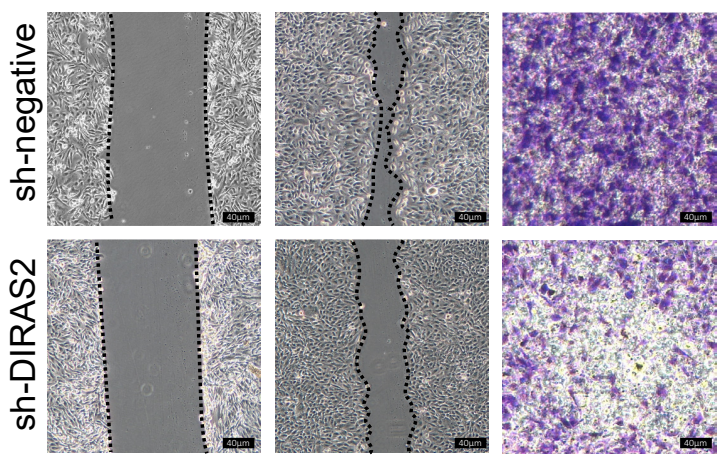

$\mathrm{Oh}$

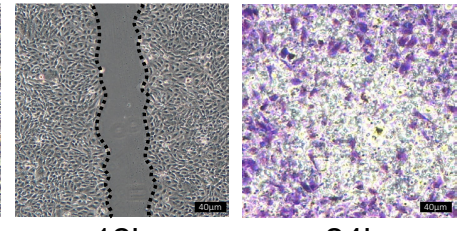

$12 \mathrm{~h}$

$24 \mathrm{~h}$
G

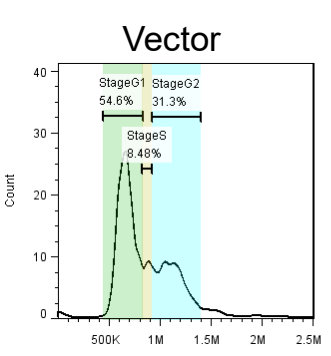

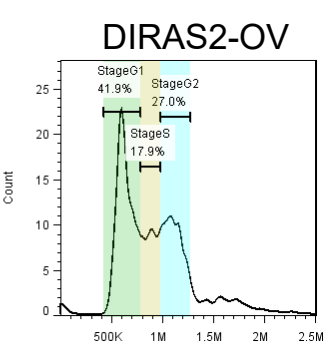

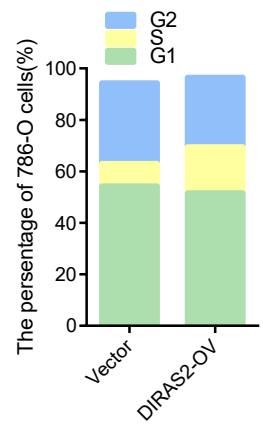

H

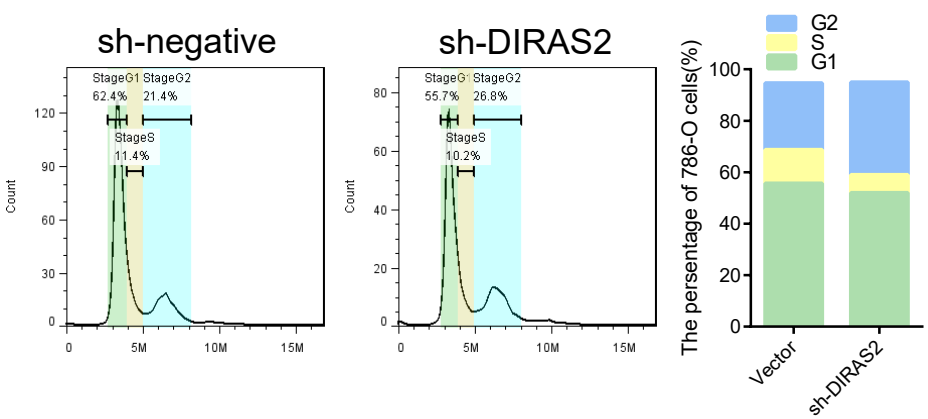


Figure 3

A

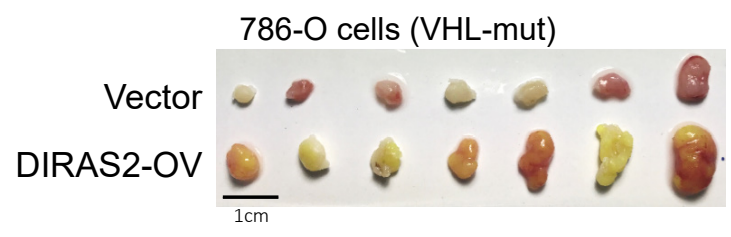

sh-negative

sh-DIRAS2

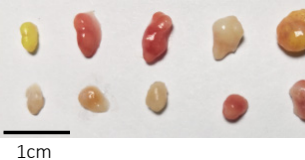

D

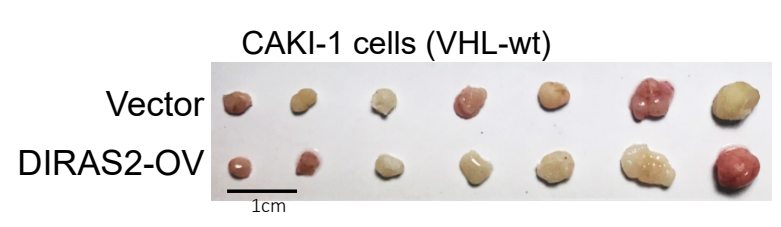

B

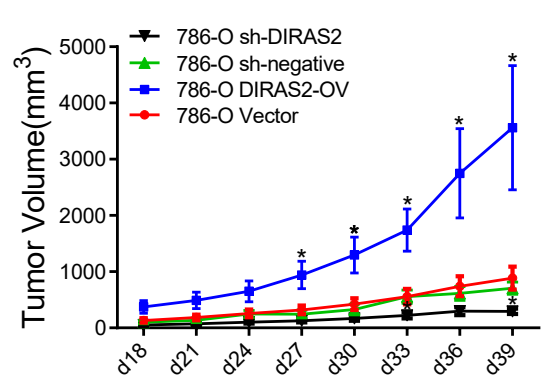

$\mathbf{E}$

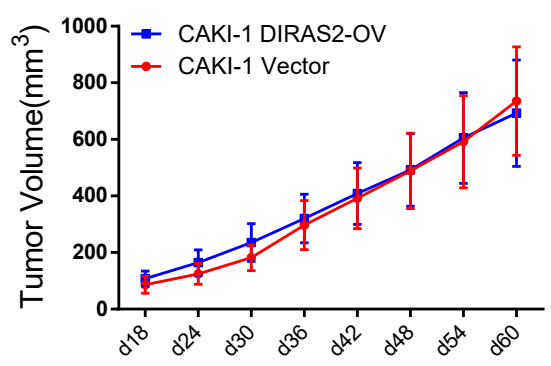

C

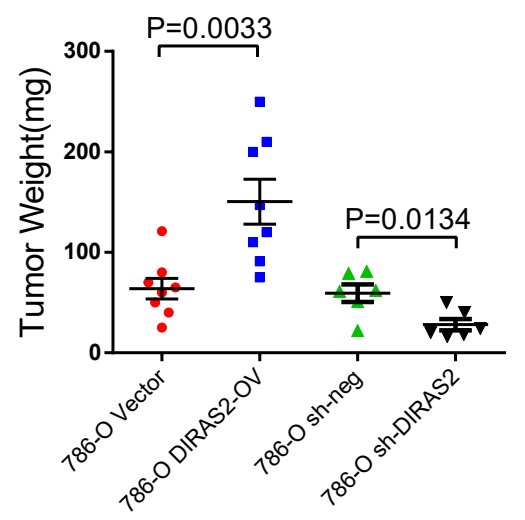

$\mathbf{F}$

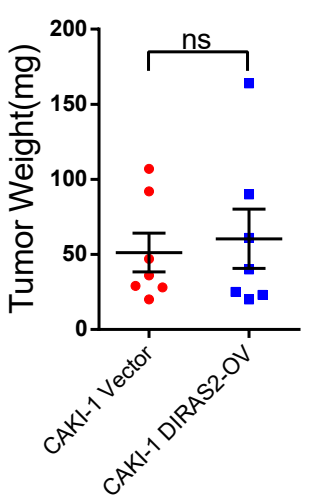


Figure 4

A

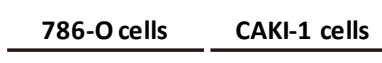

DIRAS2-Myc $(\mu \mathrm{g}) \quad 2 \quad 2 \quad 2 \quad \begin{array}{llll}2 & 2 & 2 & 2\end{array}$

Myc-tag

MAPK

PMAPK

JNK

pJNK

P38

pP38

GAPDH

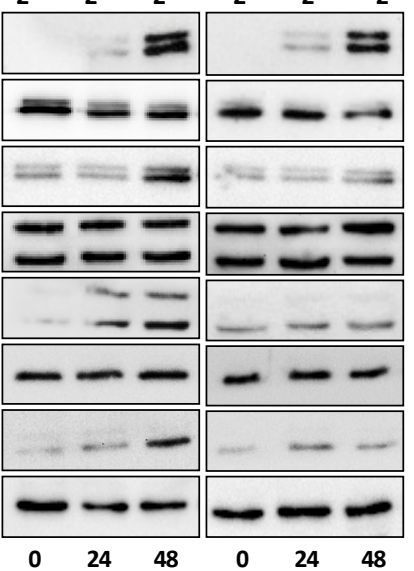

(hrs)

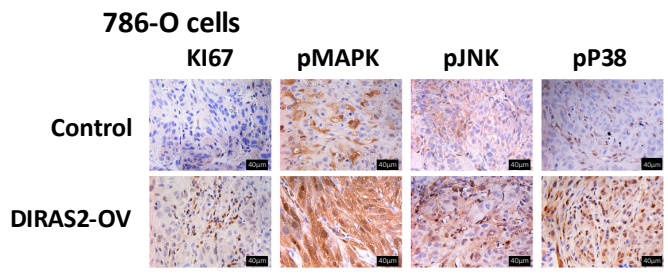

CAKI-1 cells

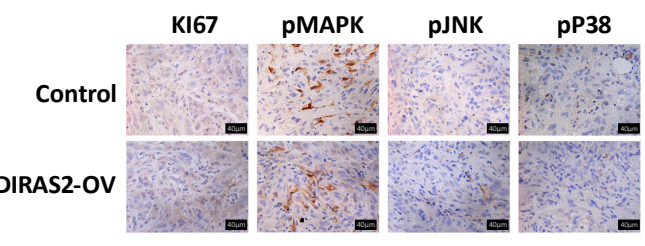

E

CAKI-1 cells

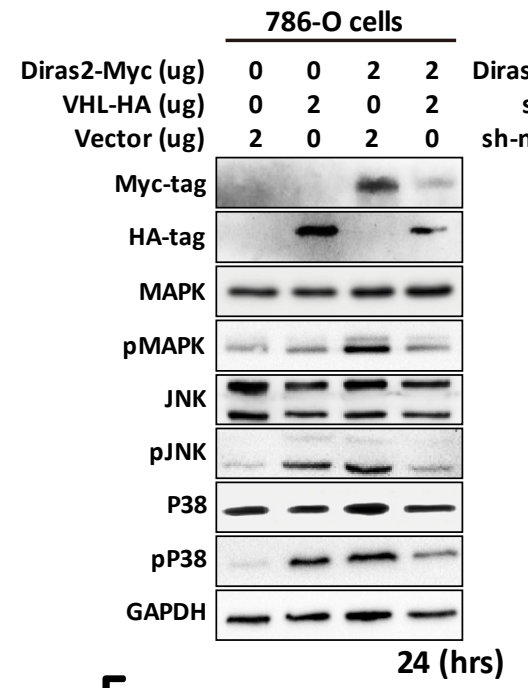

$\mathbf{F}$

293T cells

CAKI-1 cells
C

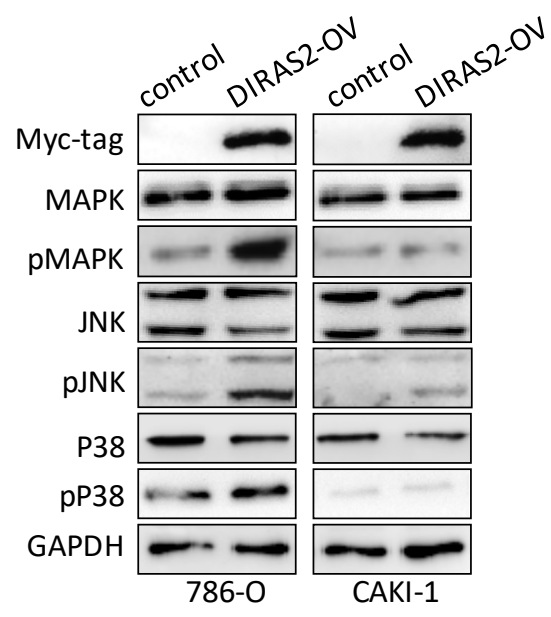

293T cells

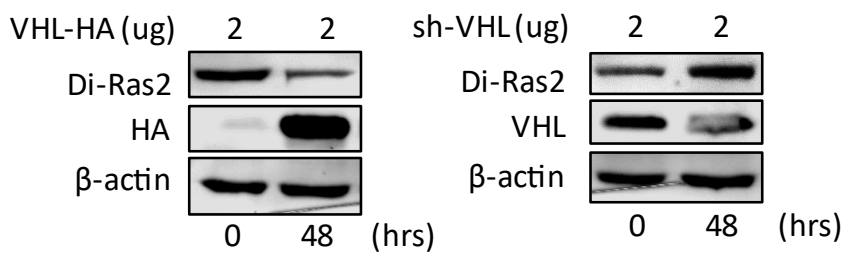

CAKI cells

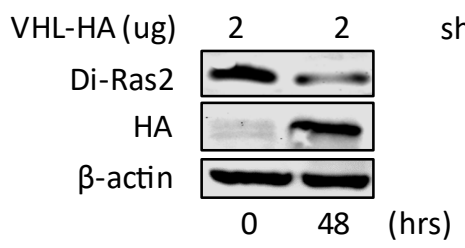

sh-VHL(ug)
Di-Ras2
VHL
$\beta$-actin

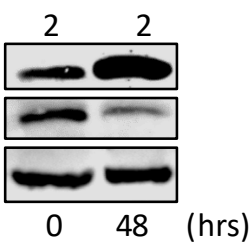

$\begin{array}{rcccccccc}\text { DIRAS2-Myc }(\mu \mathrm{g}) & 0 & 2 & 2 & 2 & 0 & 2 & 2 & 2 \\ \text { Vector }(\mu \mathrm{g}) & 0 & 0.5 & 1 & 2 & 0 & 0 & 0 & 0 \\ \text { VHL-HA }(\mu \mathrm{g}) & 0 & 0 & 0 & 0 & 0 & 0.5 & 1 & 2 \\ \text { Myc-tag } & & & & & & & & \\ \text { HA-tag } & & & & & & & \\ \text { GAPDH } & & & & \end{array}$

$\begin{array}{lllllllll}\text { DIRAS2-Myc }(\mu \mathrm{g}) & 0 & 2 & 2 & 2 & 0 & 2 & 2 & 2\end{array}$

$\begin{array}{lllllllll}\text { Vector }(\mu \mathrm{g}) & 0 & 0.5 & 1 & 2 & 0 & 0 & 0 & 0\end{array}$

$\begin{array}{lllllllll}\text { VHL-HA }(\mu \mathrm{g}) & 0 & 0 & 0 & 0 & 0 & 0.5 & 1 & 2\end{array}$

Myc-tag

HA-tag

GAPDH

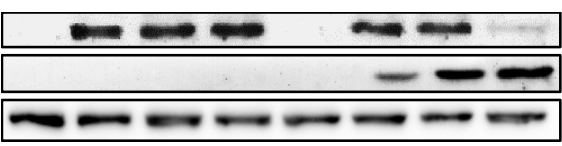

$\mathbf{G}$ 293T cells

\begin{tabular}{|c|c|c|c|c|c|c|c|c|c|c|c|c|c|}
\hline DIRAS2-Myc $(\mu \mathrm{g})$ & $\begin{array}{l}\text { T cells } \\
1.5\end{array}$ & 1.5 & 1.5 & 1.5 & 1.5 & 1.5 & $\begin{array}{r}\text { CAKI } \\
\text { DIRAS2-Myc }(\mu \mathrm{g})\end{array}$ & $\begin{array}{l}-1 \text { cel } \\
1.5\end{array}$ & 1.5 & 1.5 & 1.5 & 1.5 & 1.5 \\
\hline Vector ( $\mu \mathrm{g})$ & 1.5 & 1.5 & 1.5 & 0 & 0 & 0 & Vector $(\mu \mathrm{g})$ & 1.5 & 1.5 & 1.5 & 0 & 0 & 0 \\
\hline VHL-HA ( $\mu \mathrm{g})$ & 0 & 0 & 0 & 1.5 & 1.5 & 1.5 & VHL-HA ( $\mu \mathrm{g})$ & 0 & 0 & 0 & 1.5 & 1.5 & 1.5 \\
\hline Myc-tag & 5 & & - & 5 & - & $x^{2}$ & Myc-tag & 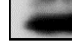 & -9 & $=$ & $\infty$ & 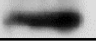 & \\
\hline GAPDH & $\longrightarrow$ & $=$ & - & $\longrightarrow$ & $=$ & 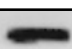 & GAPDH & c & & - & $=$ & $=$ & 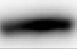 \\
\hline & 24 & 48 & 72 & 24 & 48 & 72 & (hrs) & 24 & 48 & 72 & 24 & 38 & 72 \\
\hline
\end{tabular}

CAKI-1 cells 


\section{Figure 5}

A

\section{T cells}

$\begin{array}{rcccccccc}\text { DIRAS2-Myc(ug) } & 1.5 & 1.5 & 1.5 & 1.5 & 1.5 & 1.5 & 1.5 & 1.5 \\ \text { Vector(ug) } & 1.5 & 1.5 & 1.5 & 1.5 & 0 & 0 & 0 & 0 \\ \text { VHL-HA(ug) } & 0 & 0 & 0 & 0 & 1.5 & 1.5 & 1.5 & 1.5\end{array}$

$\begin{array}{lllllllll}\text { VHL-HA(ug) } & 0 & 0 & 0 & 0 & 1.5 & 1.5 & 1.5 & 1.5\end{array}$

Myc-tag

$\beta$-actin

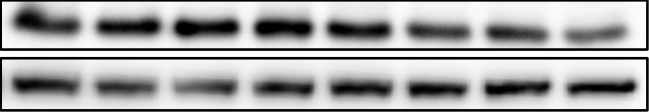

$\begin{array}{llllllllll}10 \mathrm{ug} / \mathrm{mlCHX} & 0 & 4 & 8 & 12 & 0 & 4 & 8 & 12 & \text { (hrs) }\end{array}$

B

293T cells

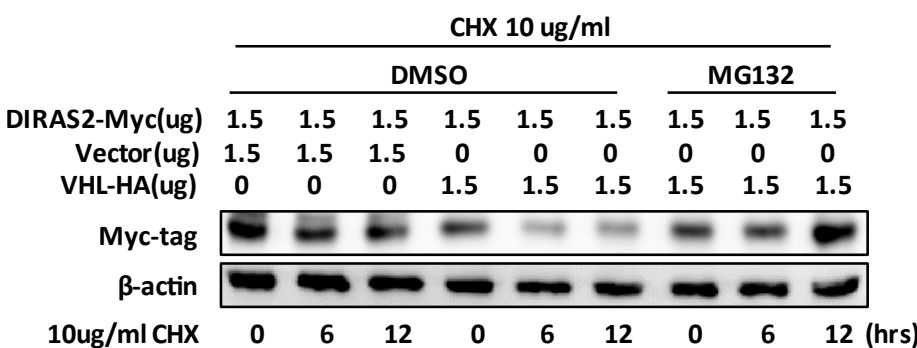

C

293T cells

IP:Myc

IB: Myc input $\overline{\lg G \quad \text { Myc }}$

IB: HA

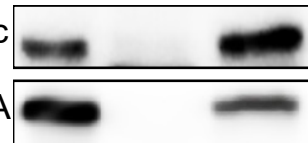

IB: HA

IB: Myc

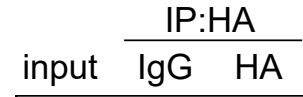

CAKI-1 cells

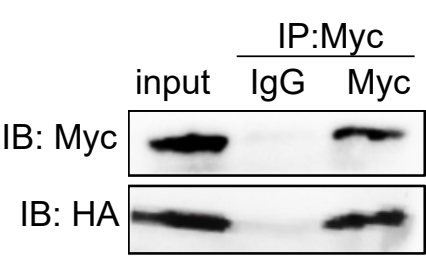

IB: Myc

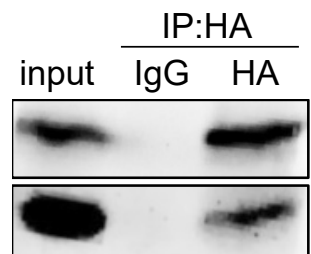

D

293T cells

IP:Di-Ras2

IB: Di-Ras2

IB: VHL input $\operatorname{lgG~Di-Ras2}$

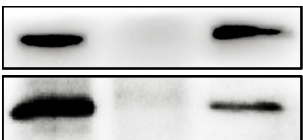

CAKI-1 cells

IP:Di-Ras2

input $\operatorname{lgG~Di-Ras2}$

IB: Di-Ras2

IB: VHL
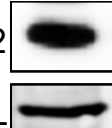

CAKI-1 cells

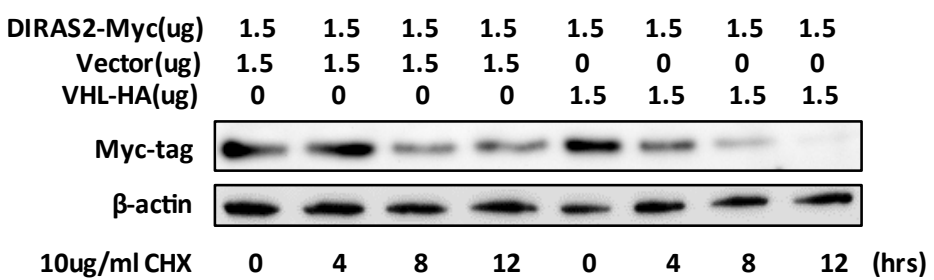

CAKI-1 cells

\begin{tabular}{|c|c|c|c|c|c|c|c|c|c|}
\hline \multirow[b]{3}{*}{ DIRAS2-Myc(ug) } & \multicolumn{9}{|c|}{$\mathrm{CHX} 10 \mathrm{ug} / \mathrm{ml}$} \\
\hline & \multicolumn{6}{|c|}{ DMSO } & \multicolumn{3}{|c|}{ MG132 } \\
\hline & 1.5 & 1.5 & 1.5 & 1.5 & 1.5 & 1.5 & 1.5 & 1.5 & 1.5 \\
\hline Vector(ug) & 1.5 & 1.5 & 1.5 & 0 & 0 & 0 & 0 & 0 & 0 \\
\hline VHL-HA(ug) & 0 & 0 & 0 & 1.5 & 1.5 & 1.5 & 1.5 & 1.5 & 1.5 \\
\hline Myc-tag & - & $=$ & $=$ & 0 & $=$ & $=$ & - & 0 & $=$ \\
\hline$\beta$-actin & - & $=$ & $=$ & - & $=$ & - & - & - & - \\
\hline 10ug/ml CHX & 0 & 6 & 12 & 0 & 6 & 12 & 0 & 6 & 12 \\
\hline
\end{tabular}

$E$

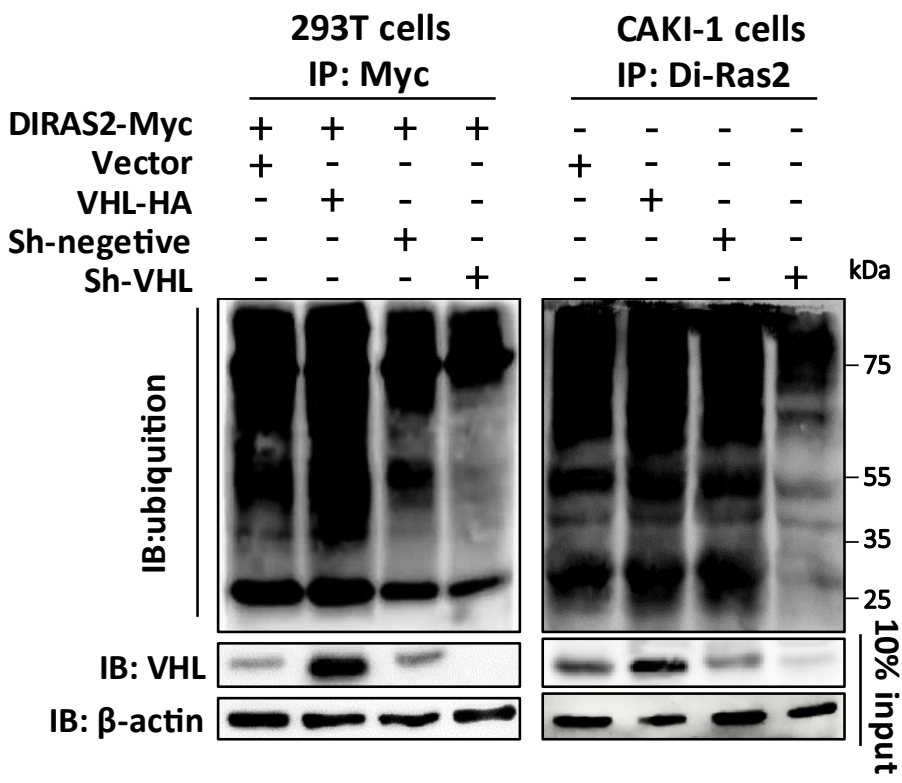

$\mathbf{F}$
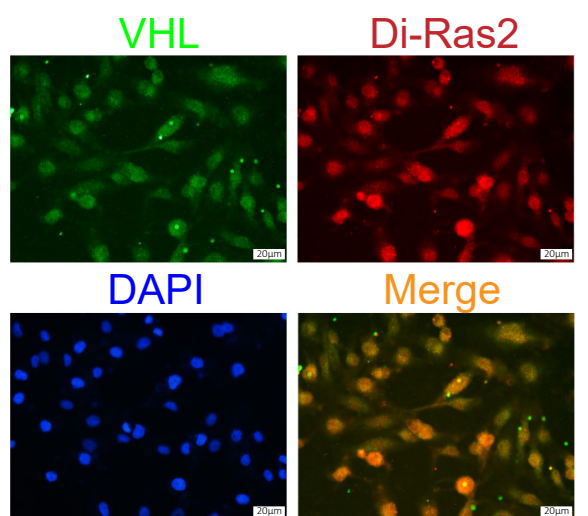

CAKI-1 cells 
Figure 6

A 786-O cells (VHL-mut)
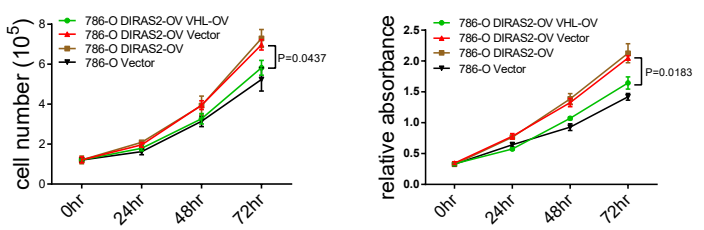

B

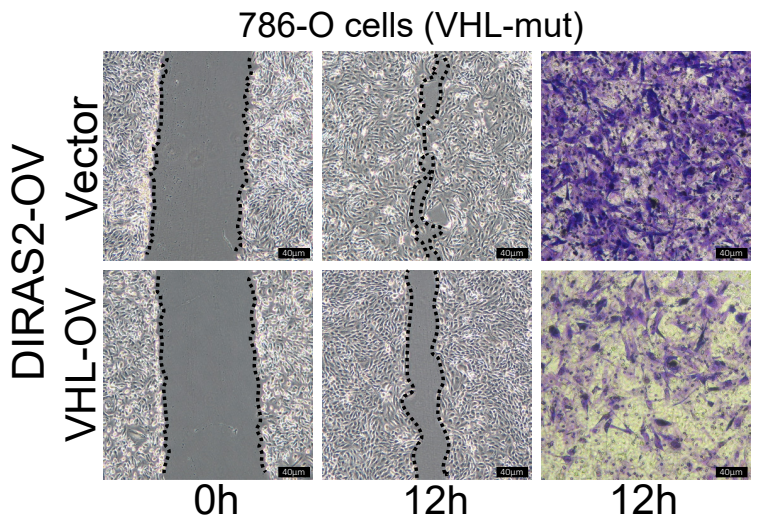

$E$

786-O cells (VHL-wt)

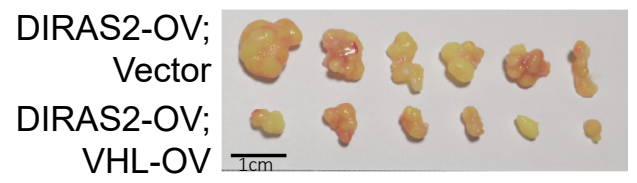

$\mathbf{F}$

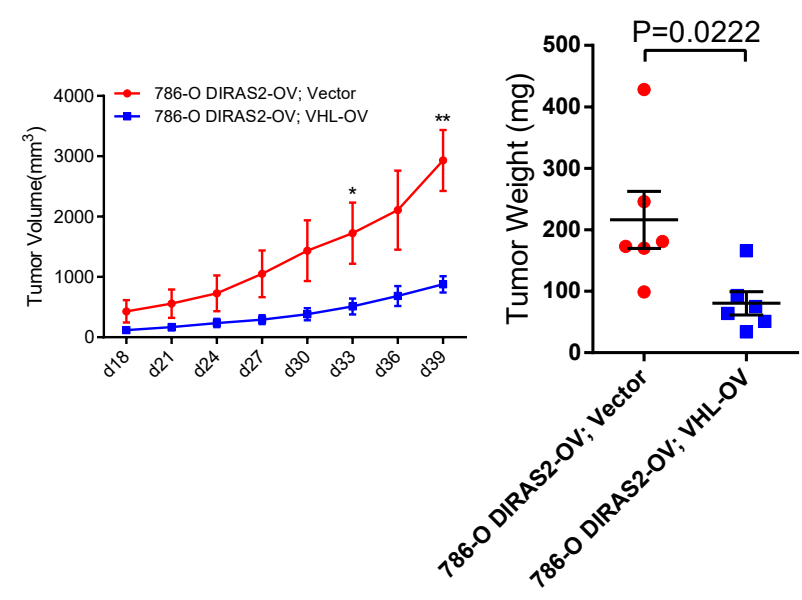

C

CAKI-1 cells (VHL-wt)
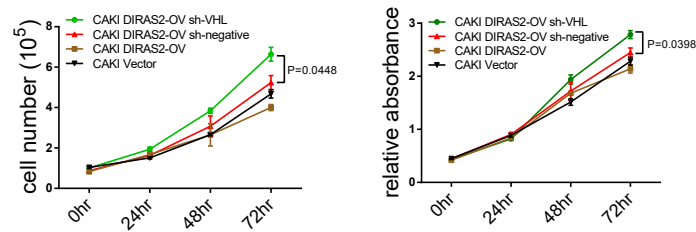

D CAKI-1 cells (VHL-wt)

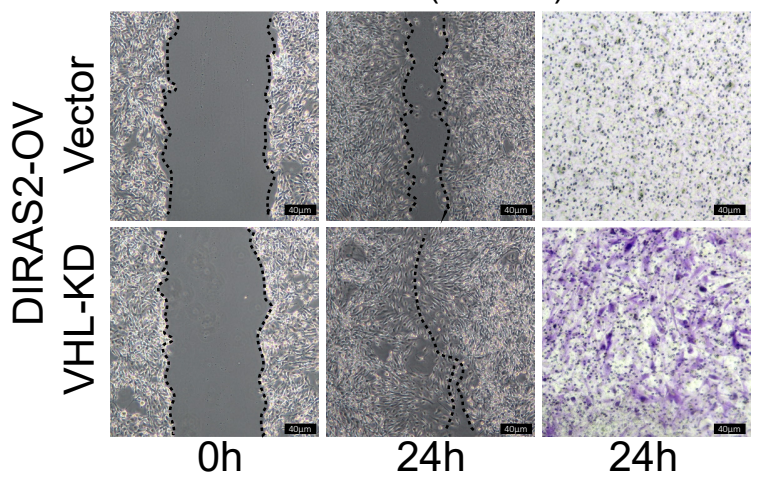

G

CAKI-1 cells (VHL-wt)

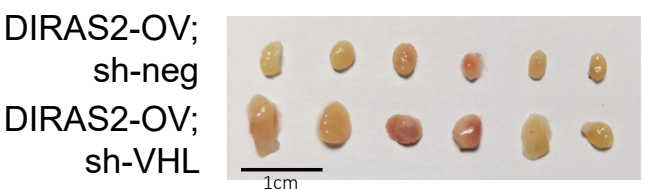

H
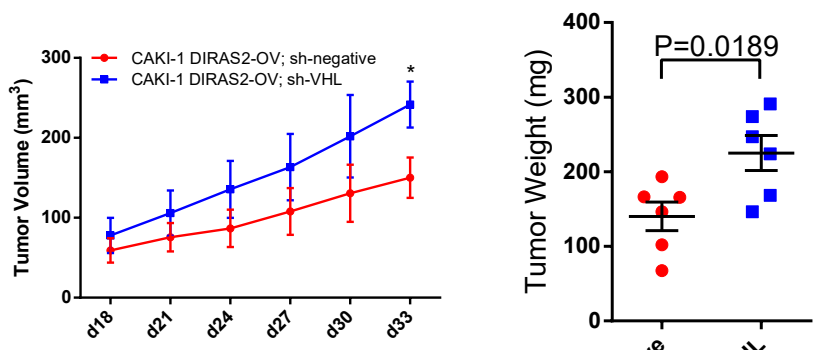
A

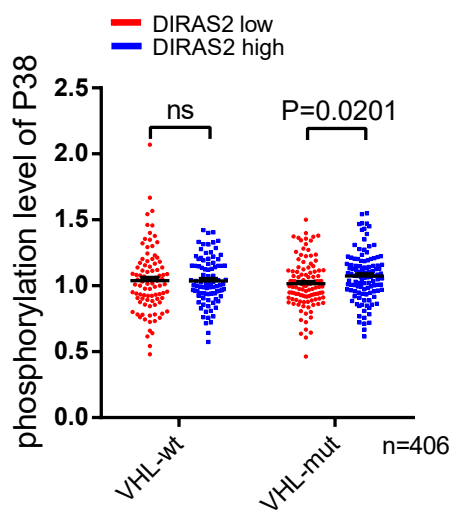

C

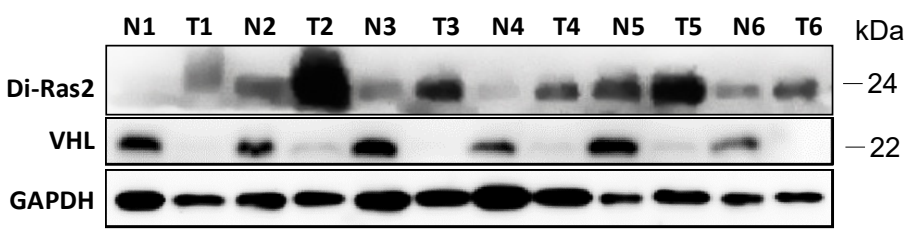

B

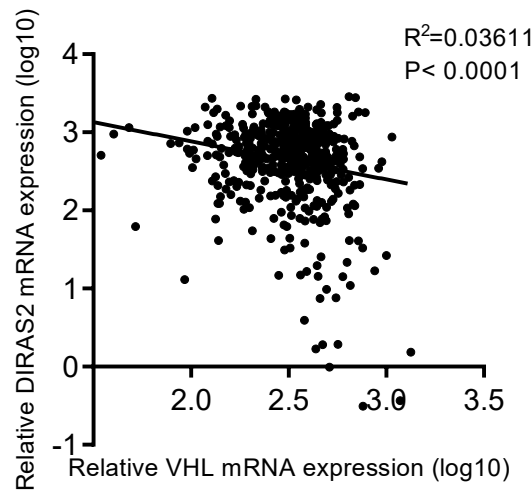

D

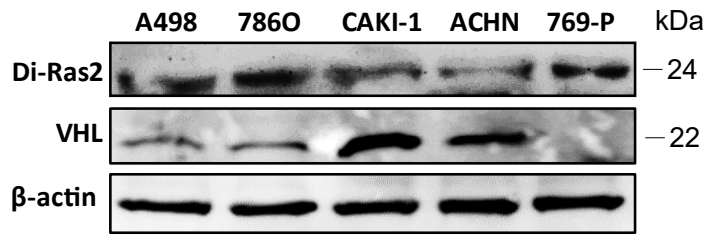

$\mathbf{E}$

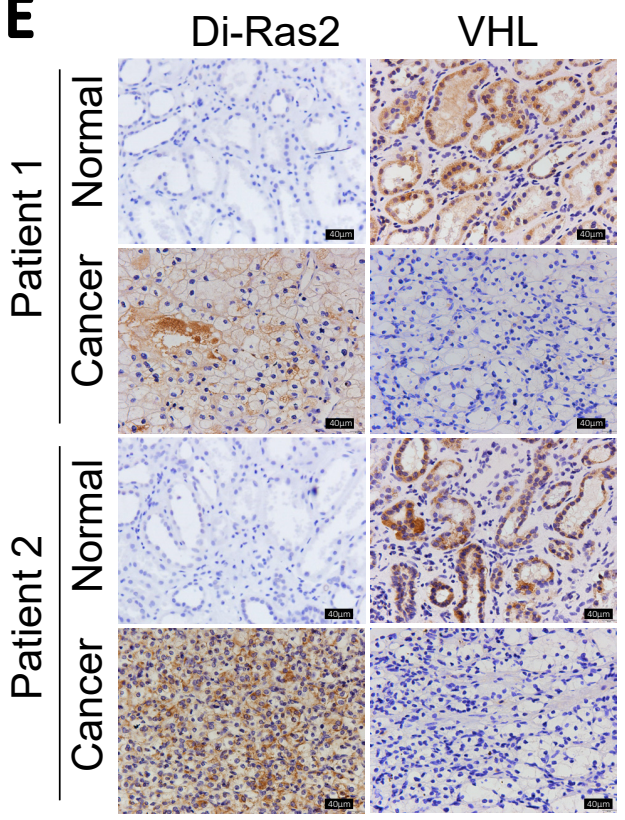

$\mathbf{F}$
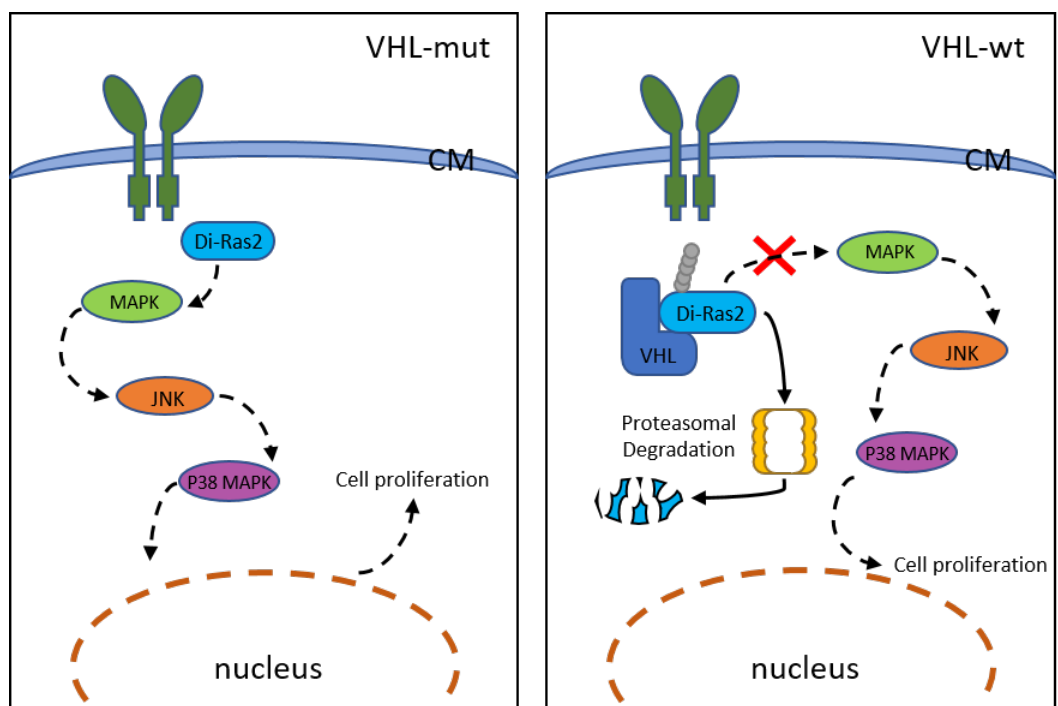
bioRxiv preprint doi: https://doi.org/10.1101/683821 this version posted June 27, 2019. The copyright holder for this preprint (which was not certified by peer review) is the author/funder. All rights reserved. No reuse allowed without permission.

Figure S1

A

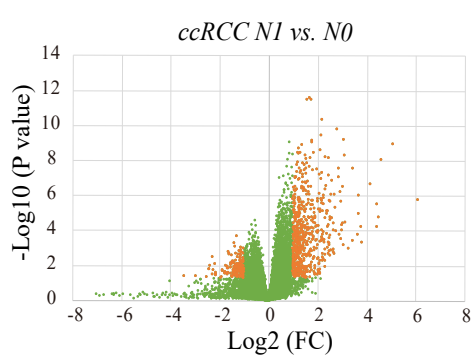

B

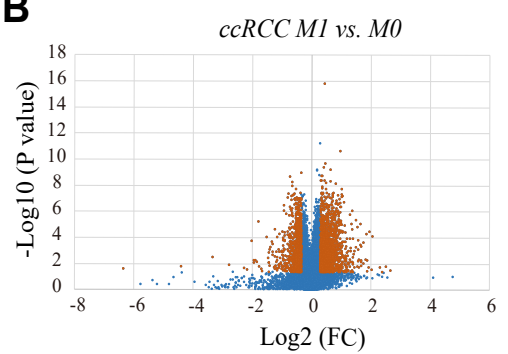

C

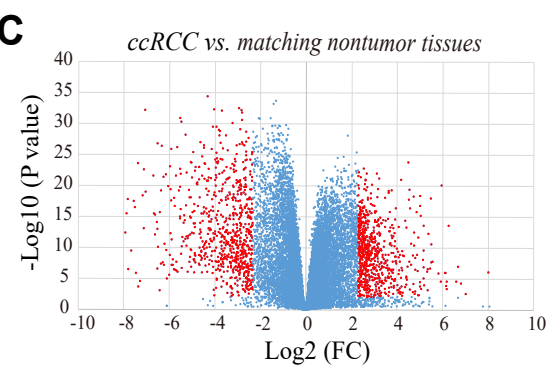

D N1 vs. No

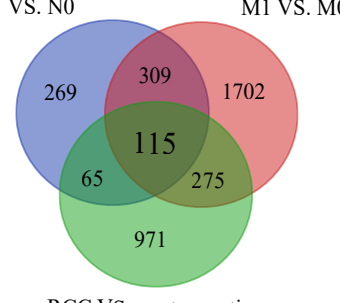

ccRCC VS. nontumor tissues

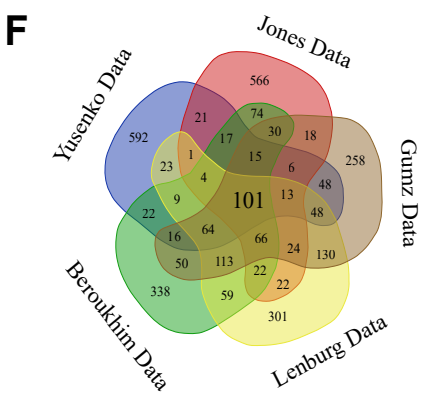

E

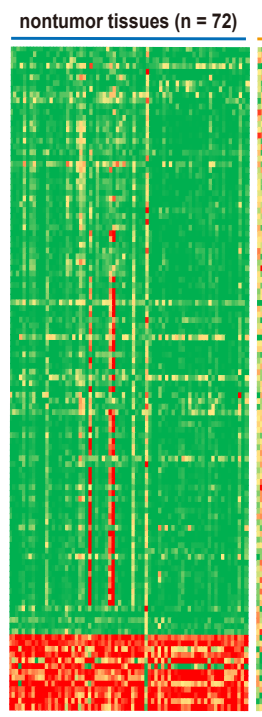

$\operatorname{ccRCC}(n=256)$

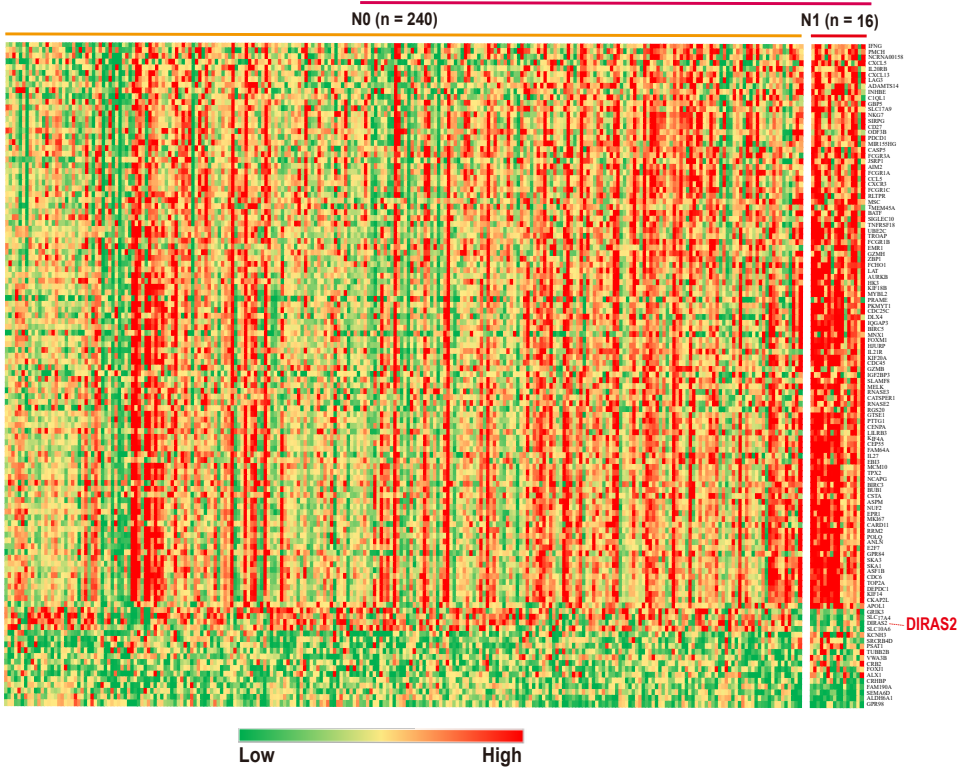


Figure S2

A

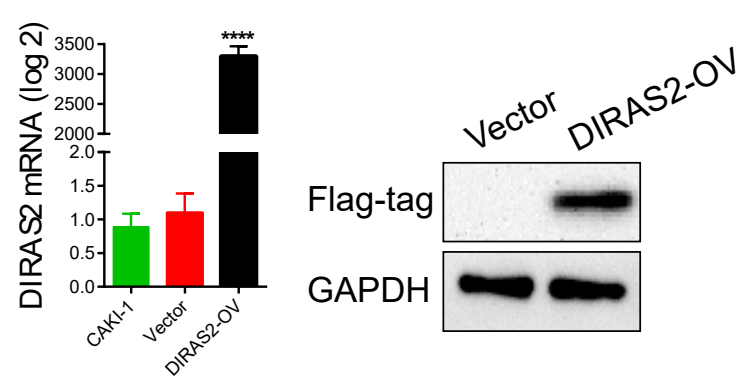

C

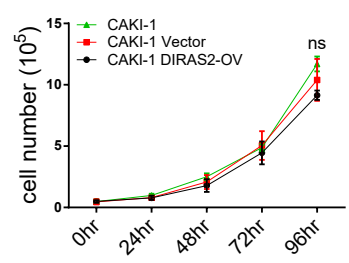

E

CAKI-1 cells (VHL-wt)

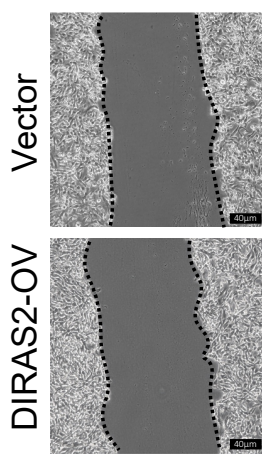

Oh
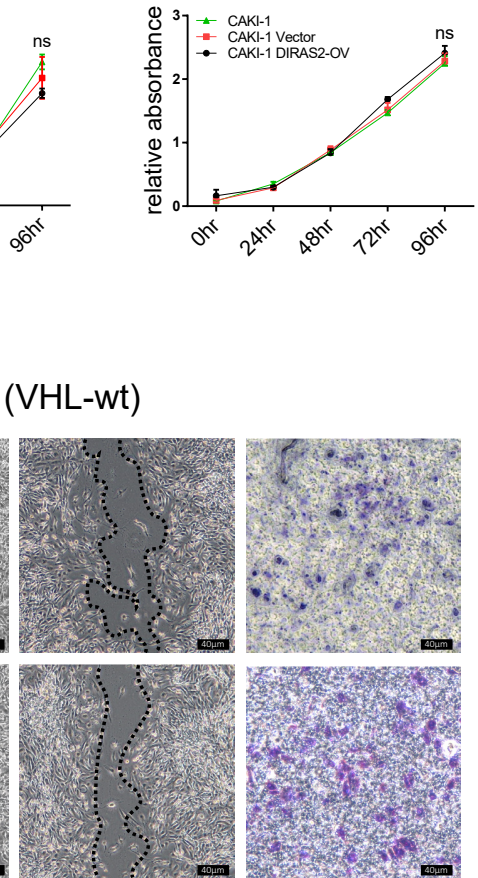

$24 \mathrm{~h}$

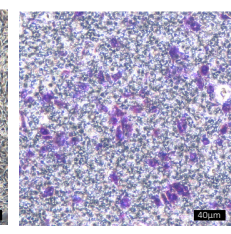

$24 h$
B

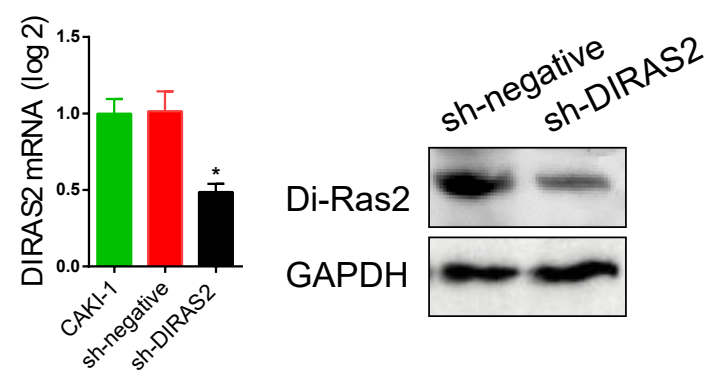

D
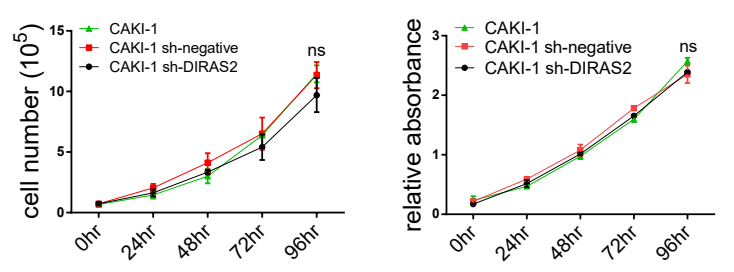

$\mathbf{F}$

CAKI-1 cells (VHL-wt)

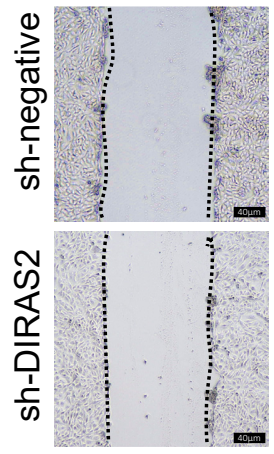

Oh

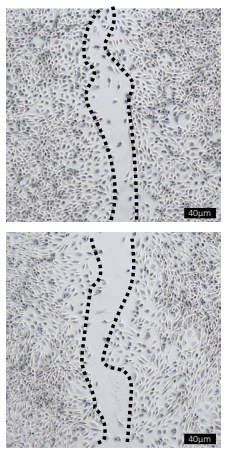

$24 \mathrm{~h}$

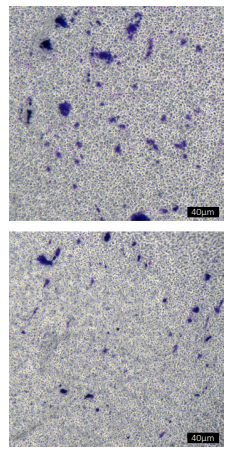

$24 \mathrm{~h}$
$\mathbf{G}$

H
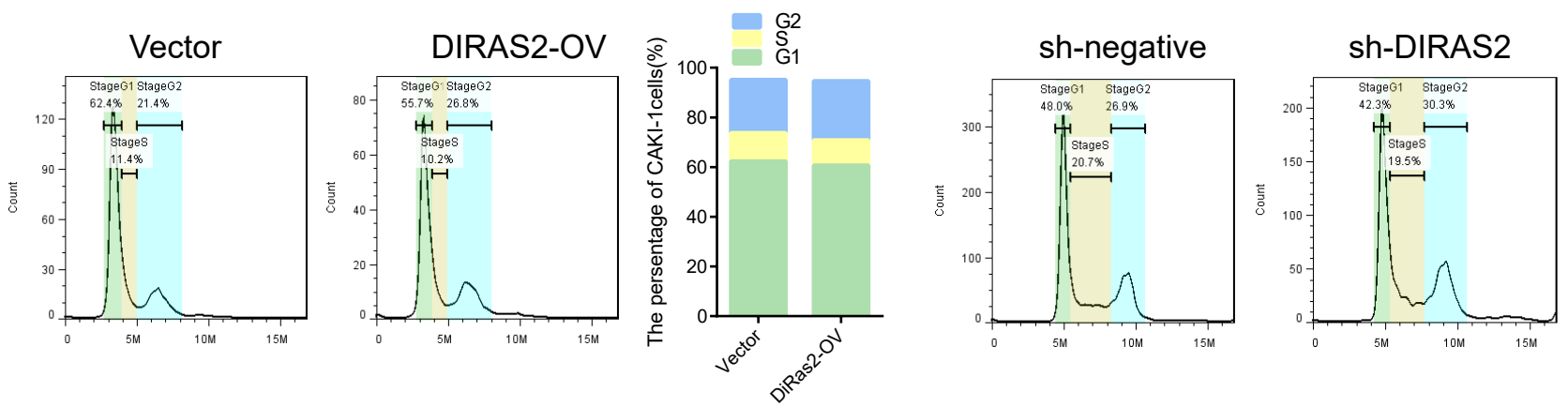

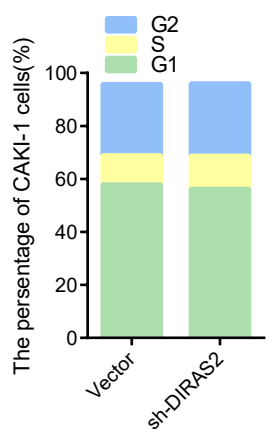


Figure S3

A

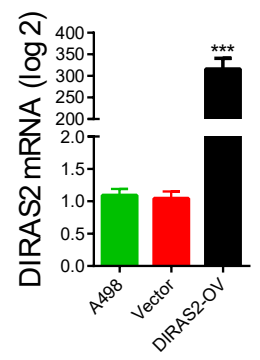

C

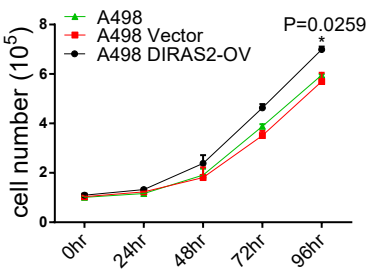

$\mathbf{E}$

A498 cells (VHL-mut)

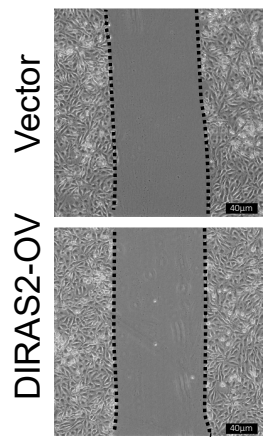

Oh

G

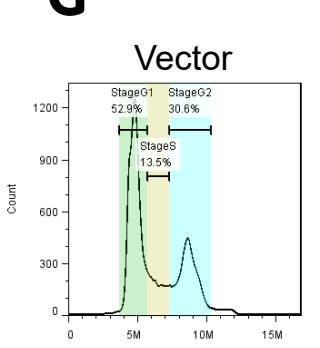

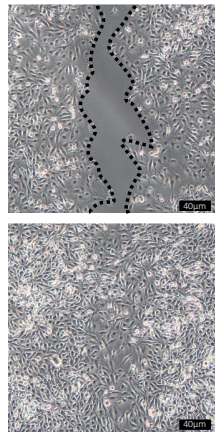

$12 \mathrm{~h}$
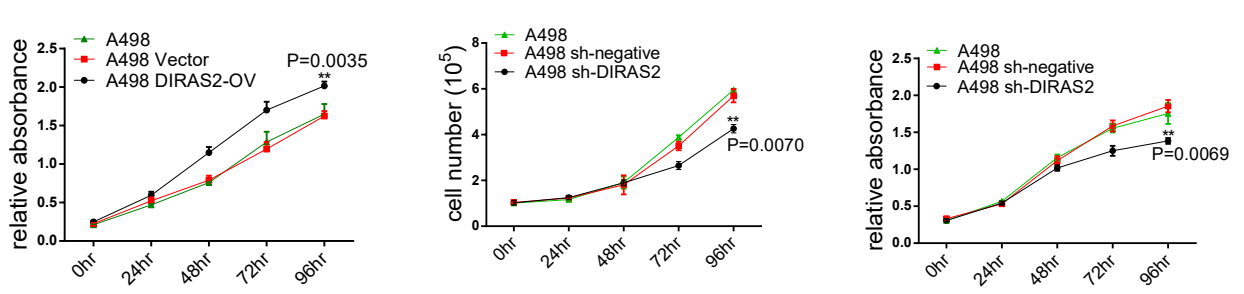

$\mathbf{F}$

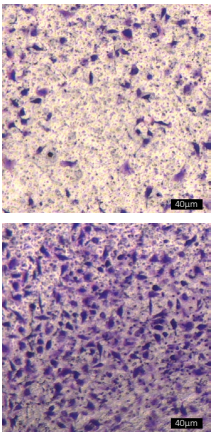

$12 \mathrm{~h}$

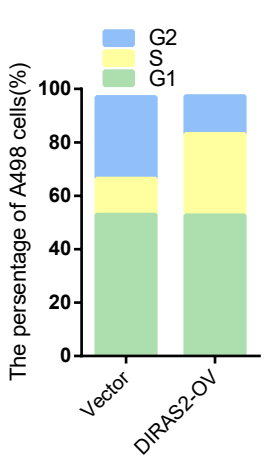

B
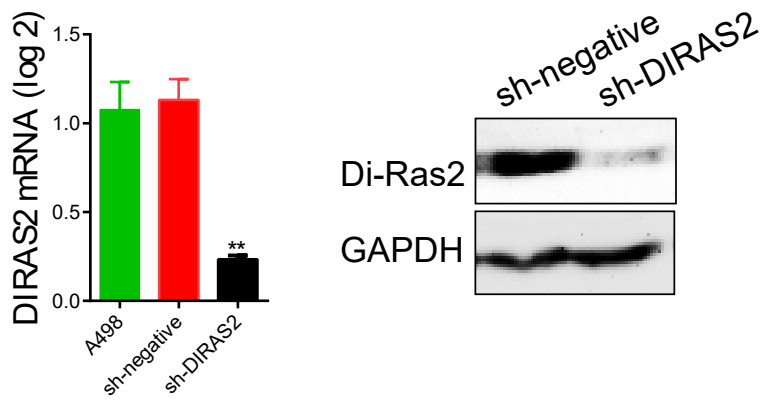

D

A498 cells (VHL-mut)

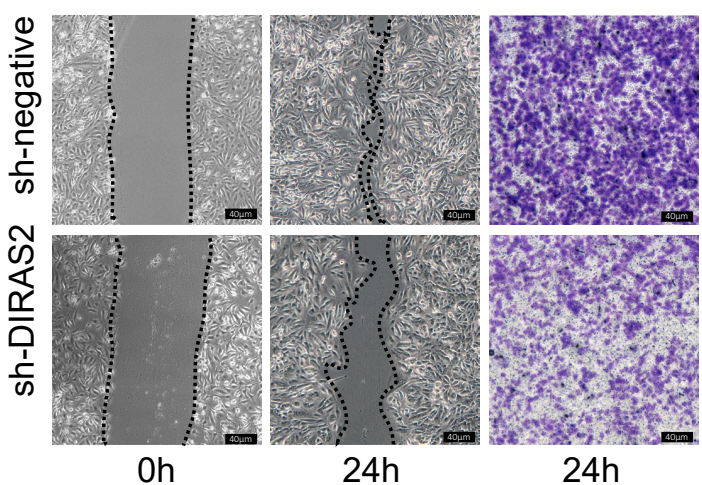

H
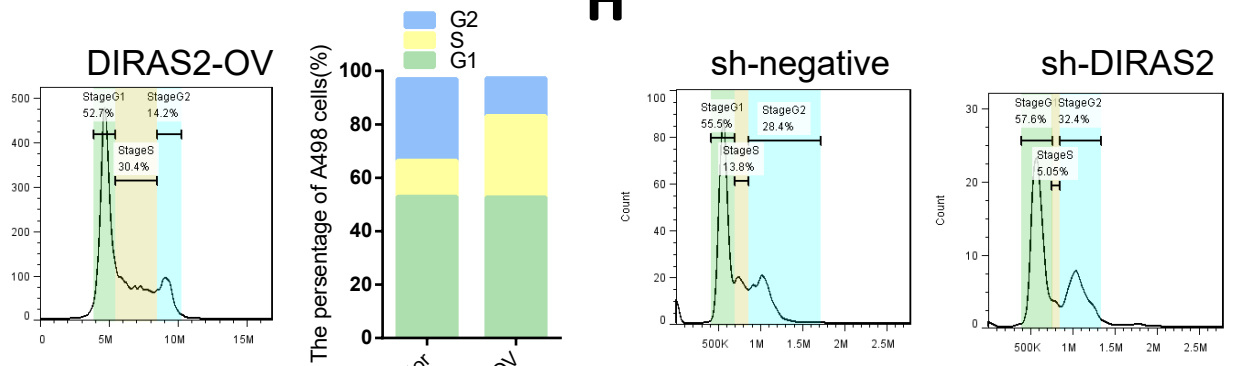

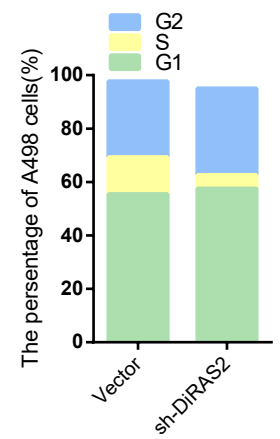


Figure S4

A

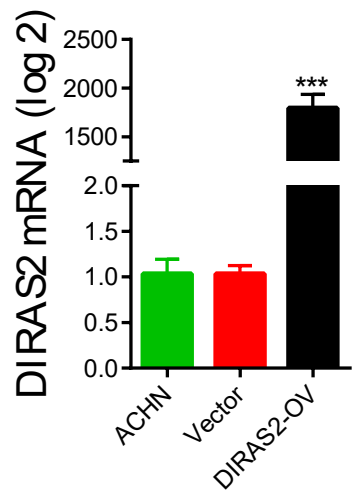

C

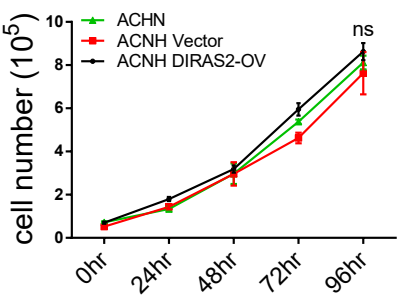

B

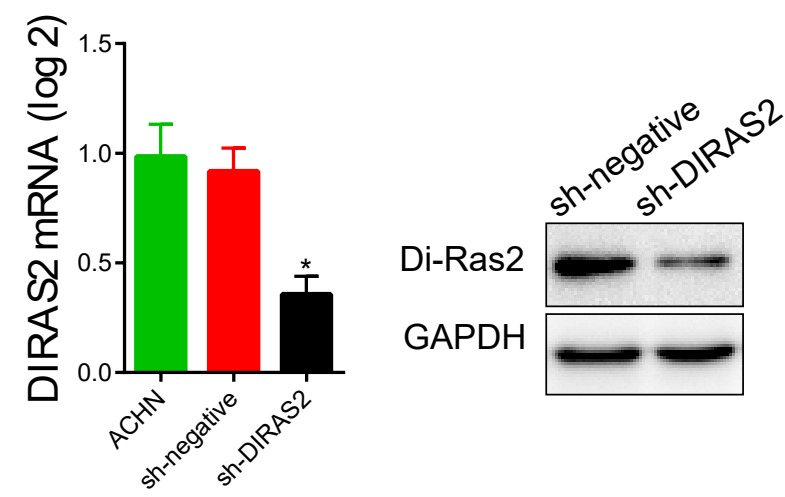

D

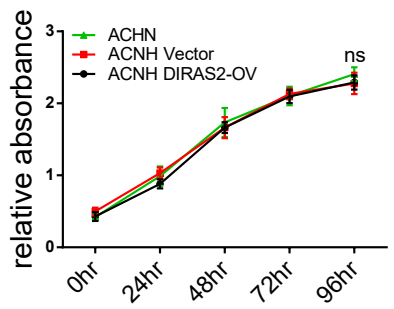

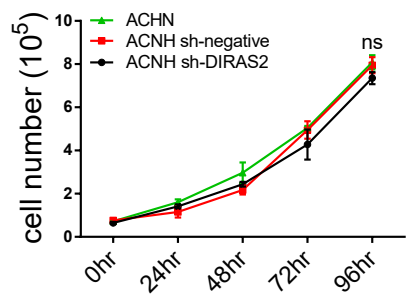

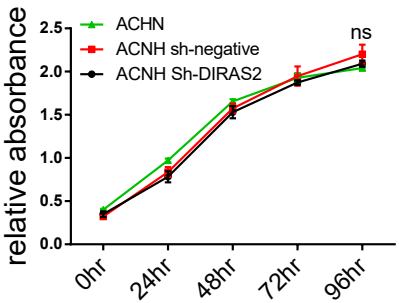


Figure S5

A

786-O cells Hypoxia

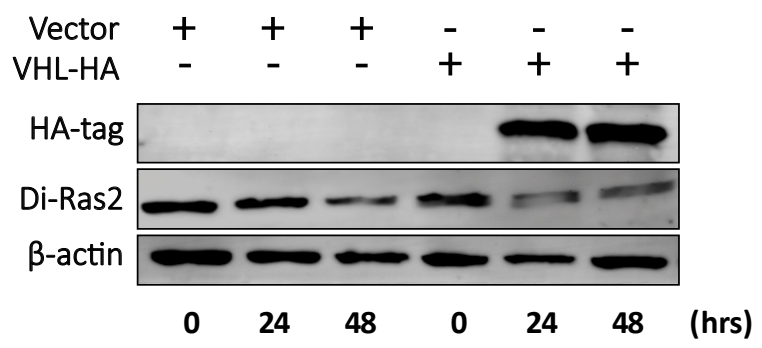

B

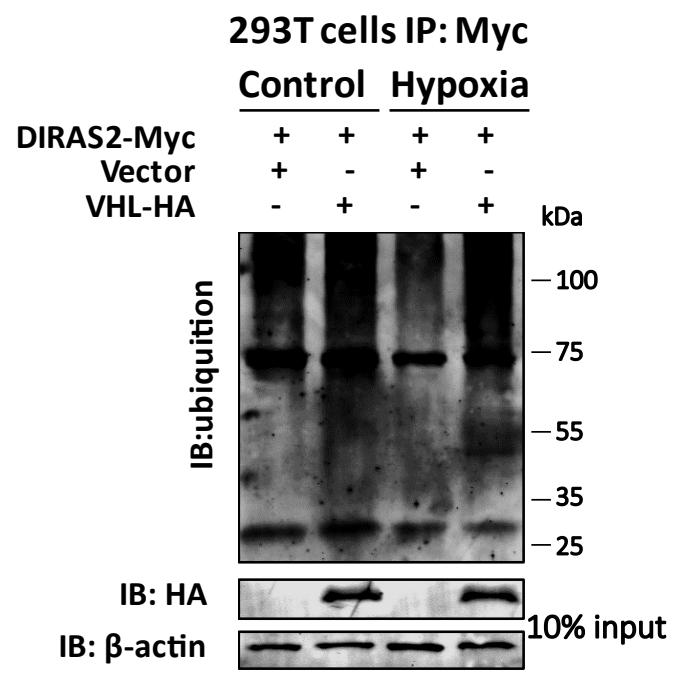

